Article

\title{
Similarity-Based Virtual Screen Using Enhanced Siamese Multi-Layer Perceptron
}

\author{
Mohammed Khaldoon Altalib ${ }^{1,2, * \mathbb{D}}$ and Naomie Salim ${ }^{1, *}$ \\ 1 School of Computing, Universiti Teknologi Malaysia, Johor Bahru 81310, Malaysia \\ 2 Computer Science Department, Education for Pure Science College, University of Mosul, Mosul 41002, Iraq \\ * Correspondence: khaldoon@graduate.utm.my (M.K.A.); naomie@utm.my (N.S.); \\ Tel.: +60-11-6384-2349 (M.K.A.)
}

Citation: Altalib, M.K.; Salim, N. Similarity-Based Virtual Screen Using Enhanced Siamese Multi-Layer Perceptron. Molecules 2021, 26, 6669. https://doi.org/10.3390/molecules 26216669

Academic Editor: Dmitri B. Kireev

Received: 17 September 2021

Accepted: 1 November 2021

Published: 3 November 2021

Publisher's Note: MDPI stays neutral with regard to jurisdictional claims in published maps and institutional affiliations.

Copyright: (c) 2021 by the authors. Licensee MDPI, Basel, Switzerland. This article is an open access article distributed under the terms and conditions of the Creative Commons Attribution (CC BY) license (https:// creativecommons.org/licenses/by/ $4.0 /)$.
Abstract: Traditional drug development is a slow and costly process that leads to the production of new drugs. Virtual screening (VS) is a computational procedure that measures the similarity of molecules as one of its primary tasks. Many techniques for capturing the biological similarity between a test compound and a known target ligand have been established in ligand-based virtual screens (LBVSs). However, despite the good performances of the above methods compared to their predecessors, especially when dealing with molecules that have structurally homogenous active elements, they are not satisfied when dealing with molecules that are structurally heterogeneous. The main aim of this study is to improve the performance of similarity searching, especially with molecules that are structurally heterogeneous. The Siamese network will be used due to its capability to deal with complicated data samples in many fields. The Siamese multi-layer perceptron architecture will be enhanced by using two similarity distance layers with one fused layer, then multiple layers will be added after the fusion layer, and then the nodes of the model that contribute less or nothing during inference according to their signal-to-noise ratio values will be pruned. Several benchmark datasets will be used, which are: the MDL Drug Data Report (MDDR-DS1, MDDR-DS2, and MDDR-DS3), the Maximum Unbiased Validation (MUV), and the Directory of Useful Decoys (DUD). The results show the outperformance of the proposed method on standard Tanimoto coefficient (TAN) and other methods. Additionally, it is possible to reduce the number of nodes in the Siamese multilayer perceptron model while still keeping the effectiveness of recall on the same level.

Keywords: drug discovery; ligand-based virtual screen; similarity model; Siamese architecture; multi-layer perceptron (MLP)

\section{Introduction}

Drug discovery is a prolonged and complex process that culminates in the manufacture of new drugs. The biomolecular target is selected, and high-performance screening procedures are executed to identify bioactive chemicals for defined aims in traditional drug research and development. It is costly and time-consuming to produce high-performing research testing [1]. In truth, the chances of success are slim; approximately 1 out of every 5000 drug candidates is expected to be accepted and widely used at some point [2]. Increased computer capabilities, on the other hand, have enabled the screening of millions of chemical compounds at a reasonable speed and cost. The virtual screening methodology is a computerized method for scanning large libraries of small compounds for the most likely structures with the goal of developing medication [3-5]. Virtual screening (VS) is used in the early stages of drug development to identify the most promising lead compounds from large chemical libraries. The development of medications has been sped up in recent years thanks to virtual screening (VS). Virtual screening is divided into two types: structure-based virtual screening (SBVS) and ligand-based virtual screening (LBVS) [6]. The SBVS approaches strategies to look for indirect chemicals that are suitable for the 
binding site of the biological target. The molecular docking technique lies at the heart of SBVS approaches [7]. On the other hand, the LBVS approach is used constantly for the prediction of molecular properties and for measuring molecular similarity because the method to represent the molecules is easy and accurate. The necessity of applications of similarity searching comes from the importance of lead optimization in drug discovery programs, in which close neighbors are looking into an initial lead compound to find decent compounds [8-10].

Modern deep learning (DL) approaches have recently been presented in a variety of fields, and they have progressed in recent years, creating a new door for researchers. The hit of DL techniques benefits from the speedy growth of the DL algorithms and the progression of high-performance computing techniques. Moreover, DL techniques have slight generalization errors, which allow them to achieve credible results on certain benchmarks or competitive tests [11,12]. In addition, the Siamese network is frequently employed to solve image and text similarity problems. It has been utilized for more complex data samples, particularly heterogeneous data samples with a variety of dimensionality and type properties $[13,14]$. However, some studies reported that pruning the parameters makes the model of deep learning smaller in size, more memory-efficient, more powerefficient, and faster at inference. The whole idea of model pruning is to reduce the number of parameters without much loss in the accuracy of the model, which means cutting down parts of the network that contribute less or nothing to the network during inference [15-17].

Various techniques have been utilized in order to augment the retrieval effectiveness of similarity search algorithms. The use of 2D similarity algorithms has gained popularity. Estimating molecular similarity is based on the assumption that structurally similar molecules seem to be more likely to have similar characteristics than structurally different ones. Therefore, the objective of similarity searching is to identify molecules that are similar in structure to the consumer's reference structures [18,19]. A number of coefficient techniques can be used to quantify the similarity/difference between molecule pairs. Many other studies tested the outcomes of various similarity coefficients, showing that the Tanimoto coefficient performed better than the others. As a result, in cheminformatics, the Tanimoto coefficient has become the most often used measure of chemical compound similarity [20-22]. Some experiments attempted to combine techniques from other fields. Such that they adapted the techniques from text information retrieval to employ in the cheminformatic domain to improve the similitude of molecular searching [23]. For example, the Bayesian inference network was based on text retrieval domains, it has been adapted and used in the similarity of molecular searches in virtual screening, and outperforms the Tanimoto technique [24,25]. Furthermore, reweighting approaches were employed to model document retrieval in the text field and modified in the cheminformatic area in the retrieval model [25-27]. Mohammed Al Dabagh (2017) improved the molecular similarity searching and molecular ranking of chemical compounds in LBVS using quantum mechanics physics theory principles [28]. Mubarak Hussien (2017) constructed a new similarity measure using current similarity measures by reweighting several bit-strings. Furthermore, the author offered ranking strategies for the development of a replacement ranking approach [29]. Deep belief networks (DBN) were used by Nasser, Majed, and colleagues (2021) to reweight molecular data wherein many descriptors were used, each reflecting separate relevant aspects, and combining all new features from all descriptors to create a new descriptor for similarity searches [30,31].

On the other hand, many studies have used deep learning methods as prediction or classification models. Some of them have used the DNN model to predict the activities of the selected compounds. Furthermore, other studies have reported that deep learning methods in Siamese architecture as a similarity model produce the best performance in many fields. For example, Jonas et al. (2016) used an LSTM Siamese neural network to calculate the similarity wherein the exponential Manhattan distance was used to measure the similarity between two sentences [32]. Jun Yu and Mengyan Li et al. (2020) used CNN Siamese architecture to determine whether two people are related, allowing missing 
persons to be reunited with their kin [33]. In the drug discovery domain, Devendra Singh Dhami et al. (2020) used images as an input in a Siamese convolutional network architecture to predict drug interactions in the drug discovery area [34]. Minji Jeon1 et al. (2019) proposed a method for calculating distance, utilizing MLP Siamese neural networks (ReSimNet) in structure-based virtual screen (SBVS) using cosine similarity [35].

Moreover, some early work in the pruning parameters domain used a gradual pruning scheme based on pruning all the weights in a layer less than some threshold (manually chosen) [12]. Blundell et al. (2015) introduced Bayes backpropagation for feedforward neural networks. This method gives the uncertainty in their predictions and reduces the model's parameter count by ordering the weights according to their signal-to-noise-ratio and setting a certain percentage of the weights with the lowest ratios to 0 to prune these weights [15]. Louizos and Christos et al. (2017) used hierarchical priors to prune nodes instead of individual weights and also used the posterior uncertainties to determine the optimal fixed-point precision to encode the weights [36]. Chenglong Zhao et al. (2019) proposed a variational Bayesian scheme for pruning convolutional neural networks at the channel level. The variational technique is introduced to estimate the distribution of a newly proposed parameter; based on this, redundant channels can be removed from the model [37].

Despite the good performances of the above methods compared to their prior, especially when dealing with molecules that have homogenous active elements structural such as classes of molecules in the MDL Drug Data Report dataset (MDDR_DR2), the performances are not satisfied when dealing with molecules with structurally heterogeneous nature such as classes of molecules in the MDL Drug Data Report dataset (MDDR_DR3, MDDR_DR1) and maximum unbiased validation (MUV) dataset. In this paper, the Siamese multi-layer perceptron model will be used and enhanced in order to achieve the main purpose of this study for improving the performance of similarity searching, especially with molecules that are structurally heterogeneous. The following are the paper's main contributions:

(1) The Siamese multi-layer perceptron will be enhanced by (a) using two distance layers and then a fuse layer that combines the results from two distance layers, with multiple layers added after the fusion layer to improve the similarity recall; (b) pruning nodes in the Siamese similarity model to reduce the number of parameters that contribute less or nothing to the network during inference.

(2) In comparison to the benchmark approach and previous studies, the suggested method outperformance in terms of results, especially when dealing with heterogeneous classes of molecules.

\section{Materials and Methods}

A Siamese neural network includes two artificial neural networks that are the same, each able to handle the hidden input data representation, which have to be linked to a final layer using a distance layer to predict whether or not two vectors fall under the same group. The networks that make up the Siamese architecture are called twins since all the weights and biases are connected. This means that both networks are symmetric. During training, the two neural networks use both feedforward perceptron and error back-propagation. As a result, it has been applied to more complex data samples, particularly heterogeneous data samples with varying dimensionality and type properties [13]. In this paper, the Siamese multilayer perceptron (MLP) model will be enhanced; the flowchart of steps for enhancing the Siamese architecture is presented in Figure 1: 


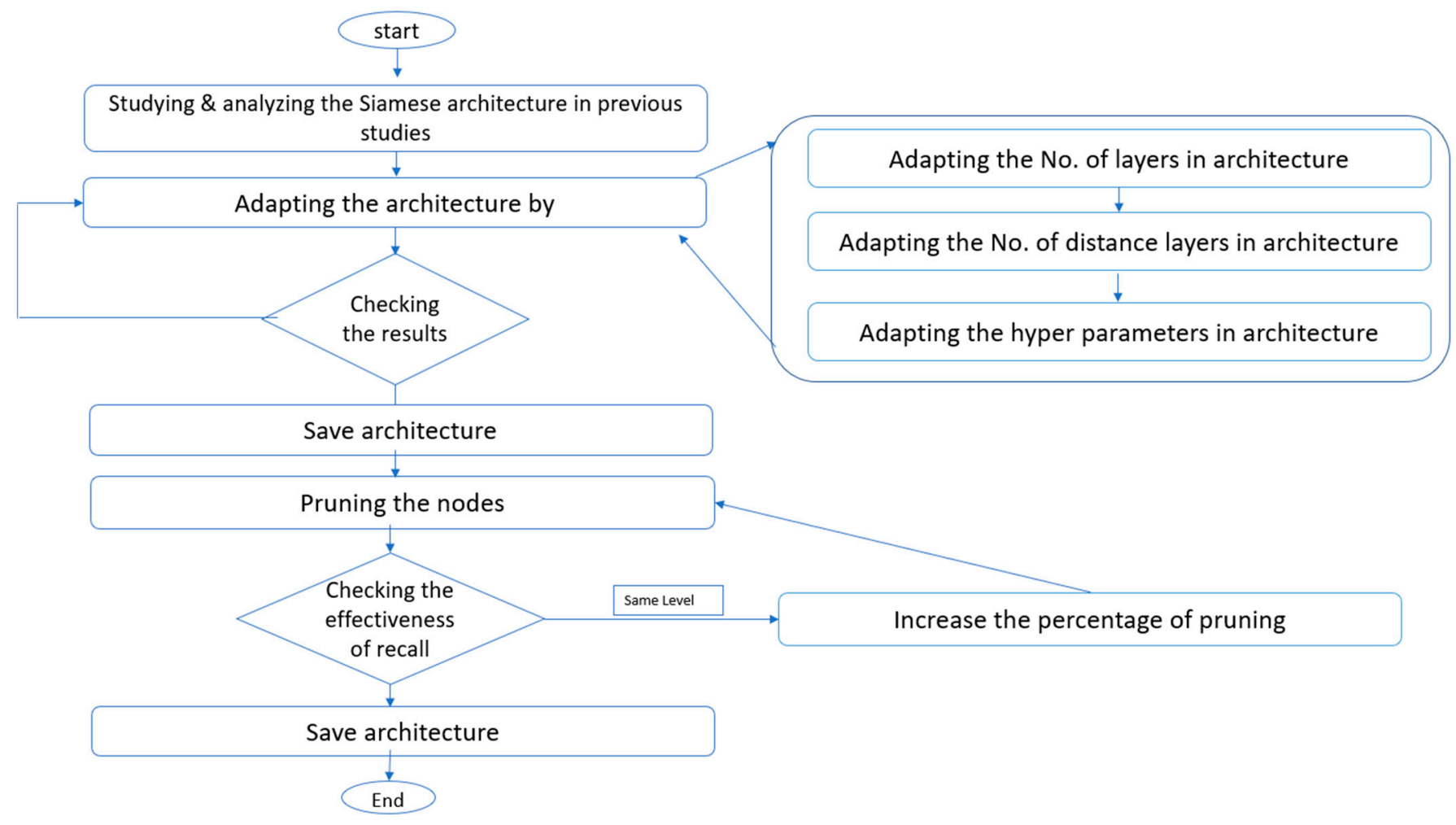

Figure 1. The flowchart of enhancing the Siamese multi-layer perceptron architecture.

The steps to enhance the Siamese architecture of the multilayer perceptron include:

(1) Many models of Siamese architecture have been studied and analyzed in various domains, such as Minji Jeon1 et al. (2019) [35], Devendra Singh Dhami et al. (2020) [34] in the field of structure-based virtual screens, and Jonas et al. (2016) in the text field [32].

(2) All prior studies used one distance layer. In this paper, two distance layers are used, and then one fusion layer combines the results from distance layers. The reason for using more than one distance layer is to further improve the similarity measurements between molecules.

(3) Many layers have been added after the fusion layer to improve the retrieval recall.

(4) To acquire a good retrieval recall outcome, the model hyperparameters, such as the number of epochs and batch size, optimization, and the activation function, have been tuned.

(5) Finally, the nodes of the model that contribute less or nothing to the network during inference are pruned without having an effect on the effectiveness of the retrieval recall.

The architecture of the Siamese MLP similarity model and the mechanism of pruning the nodes will be explained in the following subsections.

\subsection{Enhanced Siamese Multi-Layer Perceptron Similarity Model}

The architecture of the Siamese MLP similarity model consists of two inputs, representing molecular descriptors (fingerprints), and has one output that represents the degree of similarity, meaning that the output has two classes; a value of (1) means high similarity and a value of (0) means high dissimilarity. In this model, the input layer has 1024 cells, each one connected to one feature of the molecular fingerprint, with each input layer connected to distance layers. Two distances were used; the first one was the Manhattan distance, which can be represented as [38]:

$$
d_{\mathrm{AB}}=\left|f_{\mathrm{A}}-f_{\mathrm{B}}\right|
$$


$d_{\mathrm{AB}}$ : Manhatten distance

$f_{\mathrm{A}}$ : feature of molecule's query

$f_{\mathrm{B}}$ : feature of molecule's dataset

And the second distance was exponential Manhattan distance and can be given as [32]:

$$
E_{\mathrm{AB}}=\exp \left(-\left|f_{\mathrm{A}}-f_{\mathrm{B}}\right|\right)
$$

$E_{\mathrm{AB}}$ : exponential Manhatten distance

$f_{\mathrm{A}}$ : feature of molecule's query

$f_{\mathrm{B}}$ : feature of molecule's dataset

A fusion layer was then added between two distance layers-Manhattan and Exponential Manhattan - and was the reason for using more than one similarity distance to enhance the measures of similarity between molecules. The ReLU activation function has been used for all layers except the last one, in which the sigmoid activation function has been used. Moreover, the RMSprop optimizer has been used and the loss function was (binary_crossentropy); and the batch size was 256. Figure 2 demonstrates the architecture of the enhanced Siamese MLP similarity model.

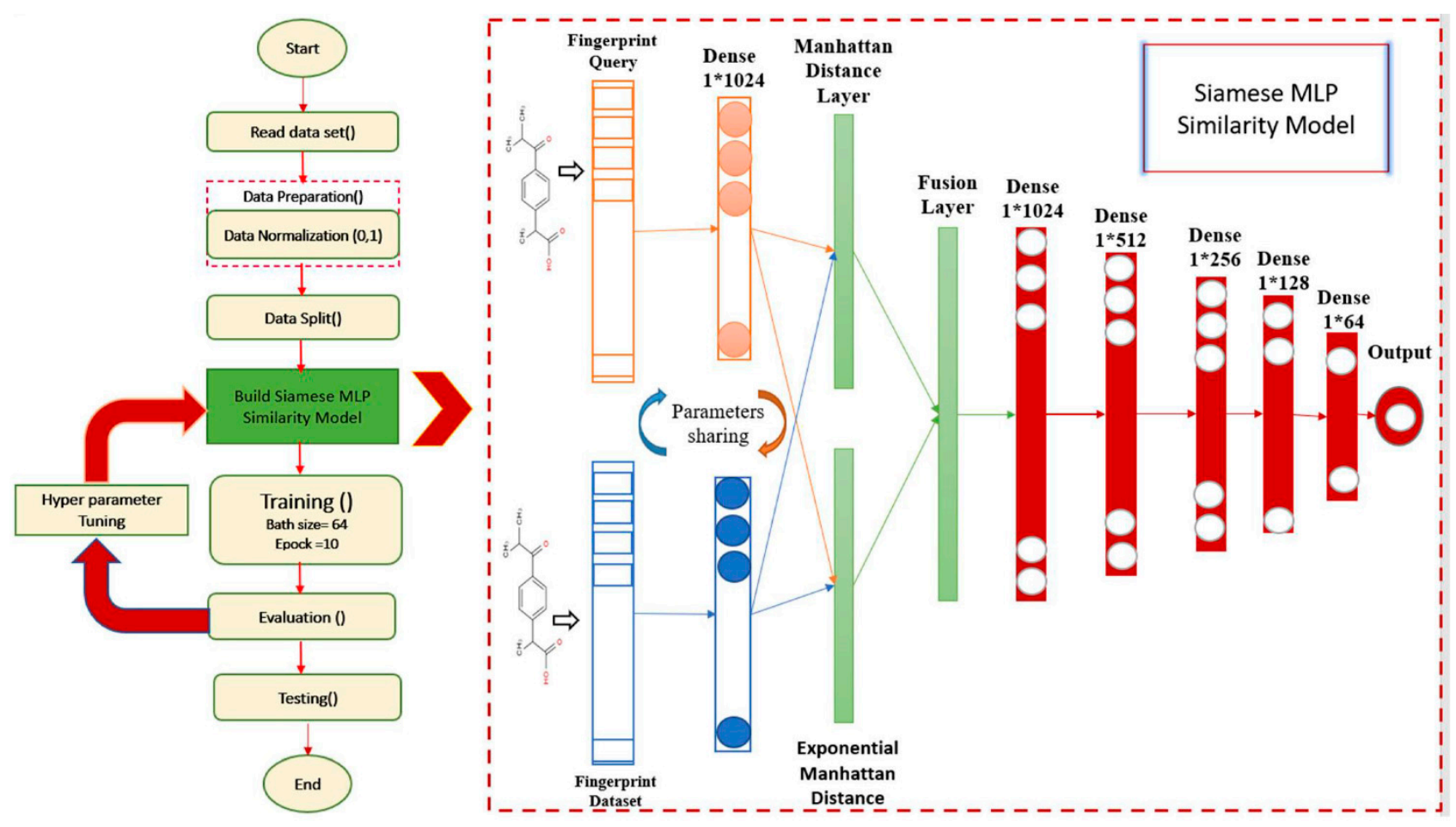

Figure 2. Enhanced Siamese MLP similarity model.

\subsection{Nodes or Neurons Pruning}

As deep neural networks contain more layers, we must multiply multiple floatingpoint integers together, which takes a long time to train and infer and consumes a lot of computing resources. The problem mentioned above can be solved in a number of ways, including weight sharing, pruning, quantization, and so on. The goal of model pruning is to reduce the number of parameters while maintaining model correctness, which entails pruning parts of the network that give little or no information to the network during inference. As a result, models are smaller in size, and more memory-efficient, power-efficient, and faster at inference with low accuracy loss [16,39]. Weight pruning and node pruning are the two most common methods of pruning. In weight pruning, individual weights are ranked in a weight matrix $\mathrm{W}$ based on their magnitude (or any other criterion), and the smallest k percent of the weights are set to zero in weight trimming. 
This corresponds to deleting connections between nodes in different layers. However, in node pruning, the columns that represented nodes in weight matrix are set to zero, in effect deleting the corresponding output neuron. Here, nodes are ranked according to their magnitude (or any other criterion), and the smallest $k$ percent of each node is set to zero. Pruning nodes will be employed in this research. Figure 3 demonstrates the idea of node pruning.
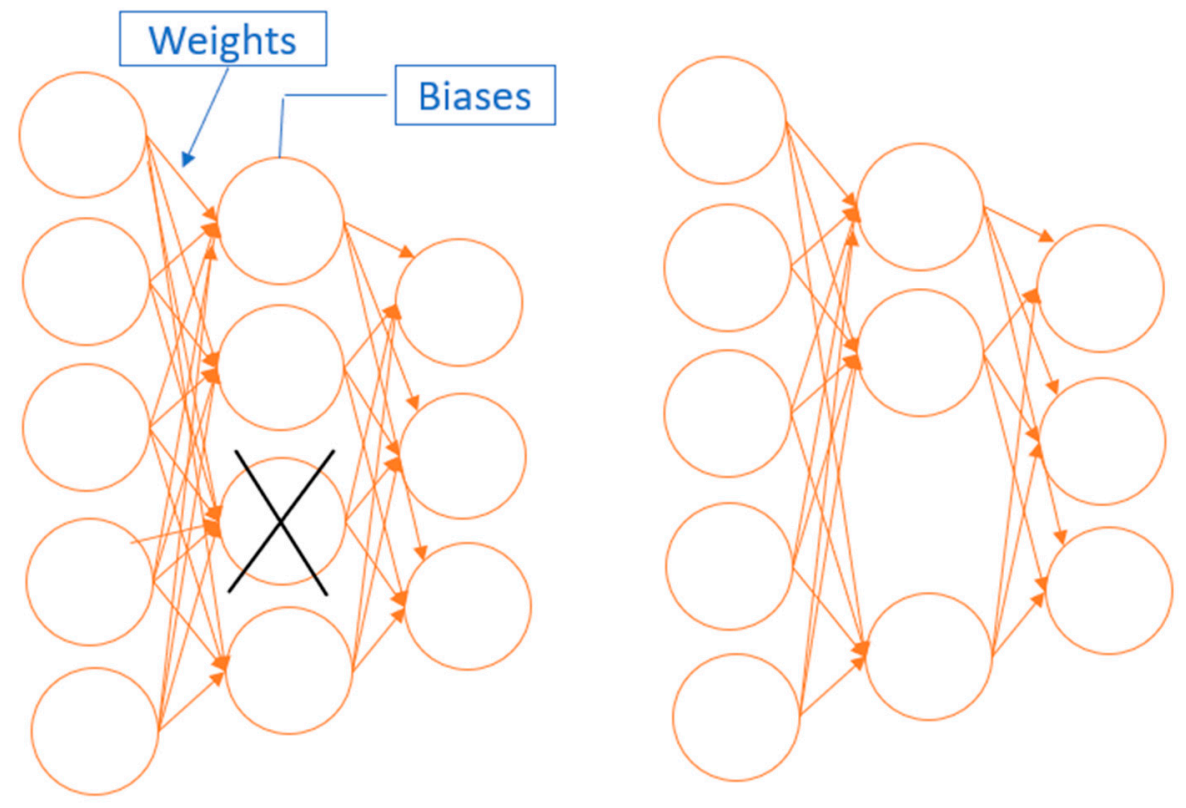

Figure 3. The idea of node pruning.

Each node is represented by a column of values in the weights matrix; the mean and variance of the column (node) are evaluated, and then the signal-to-noise ratio is calculated [15], the formula for which is:

$$
\text { signal_to_noise ratio }=\frac{\left|\mu_{i}\right|}{\sigma_{i}}
$$

$\mu$ : mean of column

$\sigma$ : variance of column

$i$ : the sequence of a column in the weight matrix

\section{Experimental Design}

\subsection{Datasets}

The MDL drug data report (MDDR) [40], maximum unbiased validation (MUV) [41], and directory of useful decoys (DUD) [42] were used in the experiments. These datasets are the most common cheminformatics datasets, and these datasets have recently been used by our study community. All molecules in the MDDR dataset were converted to fingerprints using the ECFC 4 descriptor. The screening studies were carried out with ten reference structures randomly selected from each activity class. Three 102,516-molecule datasets have been chosen (MDDR-DS1, MDDR-DS2, and MDDR-DS3). The MDDR-DS1 is divided into 11 activity groups, some of which have structurally homogeneous active elements and others which have structurally heterogeneous (i.e., structurally different) active elements. MDDR-DS2 contains ten homogeneous activity classes, whereas MDDR-DS3 contains ten heterogeneous activity classes. All of the datasets are described in Tables 1-3. Each row of a table includes the activity class, the number of molecules belonging to the class, as well as a diversity of groups, which were measured as the average similarity of Tanimoto, computed by ECFC 4 for all pairs of molecules. As shown in Table 4, Rohrer and Baumann recorded the second data collection (MUV) in this study. This data collection contains 
17 interaction groups, each of which has up to 30 active and 15,000 inactive molecules. The class composition of this dataset shows that it contains classes with a lot of variety or processes that are more heterogeneous. The last group of data used in this study is the Useful Decoys Directory (DUD), which was recently compiled for docking methods as a benchmark dataset. It was introduced by Huang et al. (2006) and was recently used in both molecular and molecular virtual screening [43]. Twelve DUD subsets with 704 active compounds and 25,828 decoys were used in this study, as shown in Table 5.

Table 1. The MDDR-DS1 structure activity classes.

\begin{tabular}{cccc}
\hline Activity Index & Active Molecules & Activity Class & Pairwise Similarity \\
\hline 31420 & 1130 & Renin inhibitors & 0.290 \\
31432 & 943 & Angiotensin II AT1 antagonists & 0.229 \\
37110 & 803 & Thrombin inhibitors & 0.180 \\
71523 & 750 & HIV protease inhibitors & 0.198 \\
42731 & 1246 & Substance P antagonists & 0.149 \\
07701 & 395 & D2 antagonists & 0.138 \\
06245 & 359 & 5HT reuptake inhibitors & 0.122 \\
78374 & 453 & Protein kinase C inhibitors & 0.120 \\
06235 & 827 & 5HT1A agonists & 0.133 \\
06233 & 752 & 5HT3 antagonist & 0.140 \\
78331 & 636 & Cyclooxygenase inhibitors & 0.108 \\
\hline
\end{tabular}

Table 2. The MDDR-DS2 structure activity classes.

\begin{tabular}{cccc}
\hline Activity Index & Active Molecules & Activity Class & Pairwise Similarity \\
\hline 07707 & 207 & Adenosine (A1) agonists & 0.229 \\
42710 & 111 & CCK agonists & 0.361 \\
31420 & 1130 & Renin inhibitors & 0.290 \\
64200 & 113 & Cephalosporins & 0.322 \\
64100 & 1346 & Monocyclic lactams & 0.336 \\
64500 & 126 & Carbapenems & 0.260 \\
64220 & 1051 & Carbacephems & 0.269 \\
75755 & 455 & Vitamin D analogous & 0.386 \\
75755 & 455 & Vitamin D analogous & 0.386 \\
07708 & 156 & Adenosine (A2) agonists & 0.305 \\
\hline
\end{tabular}

Table 3. The MDDR-DS3 structure activity classes.

\begin{tabular}{cccc}
\hline Activity Index & Active Molecules & Activity Class & Pairwise Similarity \\
\hline 09249 & 900 & Muscarinic (M1) agonists & 0.111 \\
31281 & 106 & Dopamine -hydroxylase & 0.125 \\
12464 & 505 & inhibitors & 0.102 \\
71522 & 700 & Nitric oxide synthase inhibitors & 0.103 \\
43210 & 957 & Aldose reductase inhibitors & 0.119 \\
12455 & 1400 & NMDA receptor antagonists & 0.098 \\
75721 & 636 & Aromatase inhibitors & 0.110 \\
78351 & 2111 & Lipoxygenase inhibitors & 0.113 \\
78348 & 617 & Phospholipase A2 inhibitors & 0.123 \\
78331 & 636 & Cyclooxygenase inhibitors & 0.108 \\
\hline
\end{tabular}

Table 4. MUV structure activity classes.

\begin{tabular}{cccc}
\hline Activity Index & Active Molecules & Activity Class & Pairwise Similarity \\
\hline 66 & 30 & S1P1 rec. (agonists) & 0.117 \\
644 & 30 & Rho-Kinase2 (inhibitors) & 0.122 \\
\hline
\end{tabular}


Table 4. Cont.

\begin{tabular}{cccc}
\hline Activity Index & Active Molecules & Activity Class & Pairwise Similarity \\
\hline 600 & 30 & SF1 (inhibitors) & 0.123 \\
689 & 30 & Eph rec. A4 (inhibitors) & 0.113 \\
652 & 30 & HIV RT-RNase (inhibitors) & 0.099 \\
712 & 30 & HSP 90 (inhibitors) 30 & 0.106 \\
692 & 30 & SF1 (agonists) & 0.114 \\
733 & 30 & ER-b-Coact. Bind. (inhibitors) & 0.114 \\
713 & 30 & ER-a-Coact. Bind. (inhibitors) & 0.113 \\
810 & 30 & FAK (inhibitors) & 0.107 \\
737 & 30 & ER-a-Coact. Bind. (potentiators) & 0.129 \\
846 & 30 & FXIa (inhibitors) & 0.161 \\
832 & 30 & Cathepsin G (inhibitors) & 0.151 \\
858 & 30 & D1 rec. (allosteric modulators) & 0.111 \\
852 & 30 & FXIIa (inhibitors) & 0.150 \\
548 & 30 & PKA (inhibitors) & 0.128 \\
859 & 30 & M1 rec. (allosteric inhibitors) & 0.126 \\
\hline
\end{tabular}

Table 5. DUD structure activity classes, where $\mathrm{N}_{\mathrm{a}}$ denotes the number of active compounds, and $\mathrm{N}_{\mathrm{dec}}$ denotes the number of decoys.

\begin{tabular}{cccc}
\hline No. & Dataset & \multicolumn{2}{c}{ Active and Inactive } \\
& & $\mathbf{N}_{\mathbf{d e c}}$ & $\mathbf{N}_{\mathbf{a}}$ \\
\hline 1 & FGFR1T & 4550 & 120 \\
2 & FXA & 5745 & 146 \\
3 & GART & 879 & 40 \\
4 & GBP & 2140 & 52 \\
5 & GR & 2947 & 78 \\
6 & HIVPR & 2038 & 62 \\
7 & HIVRT & 1519 & 43 \\
8 & HMGA & 1480 & 35 \\
9 & HSP90 & 979 & 37 \\
10 & MR & 636 & 15 \\
11 & NA & 1874 & 49 \\
12 & PR & 1041 & 27 \\
\hline total & - & 25,828 & 704 \\
\hline
\end{tabular}

\subsection{Evaluation Measures of the Performance}

The following criteria are used to assess the efficacy of the suggested method:

1. The first method is to look for active chemical compounds in the top $1 \%$ and $5 \%$ of the scored test set and calculate the recall value. This metric has been employed in a number of previous approaches $[27,28,30,31,44-48]$.

2. Comparison method: the second method is to compare current techniques that may be utilized to evaluate the proposed model's findings. These techniques include the following:

(a) TAN: the Tanimoto similarity coefficient has been the search benchmark method in LBVS for many years. The Tanimoto coefficient is used in its continuous form for similarities. It has been utilized in the datasets DS1, DS2, DS3, MUV, and DUD.

(b) BIN: the second technique is the Bayesian inference network, which used the ECFC4 descriptors in datasets DS1, DS2, DS3, and MUV. This is another way of comparing the results in the similarity model of molecular fingerprints in LBVS [24].

(c) SQB: the third method is quantum similarity search SQB in the MDDR dataset (DS1, DS2, DS3, and MUV) for the ECFC4 descriptor. This method utilizes a quantum mechanics approach as the model of similarity searching in LBVS [28]. 
(d) SDBN: the last technique is the deep belief networks, used to reweight the chemical characteristics, where ECFC-4, EPFP-4, and ECFP-4 descriptors were analyzed using the stack of deep belief networks technique on the MDDR dataset (DS1, DS2, DS3) [31].

3. The Kendall $\mathrm{W}$ concordance test is another important metric that could be used to measure the performance of the suggested techniques and rank the similarity methods. The concordance coefficient is a measure of agreement among raters. In the Kendall $\mathrm{W}$ test, each case represents a judge or rater, while each variable represents the thing or person being assessed. The domain of a Kendall $\mathrm{W}$ test score is between 0 and 1 . If the test score is 0 , this means no agreement; if the test score 1 , this means complete agreement. Assume the object (i) is considered as the similarity method, (ranked objects) is given the rank $r_{i j}$ by the raters $j$ (activity class), where there are in total $(n)$ objects and $(m)$ raters. Then, the total rank $(R)$ given to object (i) is [49]:

$$
\Re_{i}=\sum_{j=i}^{m} r_{i j}
$$

Then, the mean value $(\hat{R})$ is calculated by these total rankings as:

$$
\bar{\Re}=\frac{1}{2} m(n+1)
$$

Then, the sum of squared deviations $(\delta)$ is calculated as:

$$
\delta=\sum_{i=i}^{n}\left(\Re_{i}-\bar{\Re}\right)^{2}
$$

Then, the Kendall $\mathrm{W}$ test is calculated as:

$$
W=\frac{12 \delta}{m^{2}\left(n^{3}-n\right)}
$$

The results of this test are the Kendall coefficient (between 0 and 1 ) and significance level ( $p$-value); if the $p$-value is less than 0.05 , the result is considered significant, and the similarity methods can be ranked.

\section{Results and Discussion}

The experimental results from the MDDR-DS1, MDDR-DS2, MDDR-DS3, MUV, and DUD datasets, for the ECFC-4 descriptor, are provided in Tables 6-15, respectively, using $1 \%$ and $5 \%$ cut-offs. These tables show the results of the enhanced Siamese MLP similarity model compared to the benchmark TAN, as well as earlier studies BIN, SQB, and SDBN for MDDR datasets, BIN and SQB for MUV datasets, and SQB for DUD datasets. Every row in the tables displays the recall for the top $1 \%$ and $5 \%$ of the activity class, with the best recall rate shaded in each row. The mean rows in the tables represent the average for all activity classes, whereas the rows with shaded cells represent the total number of shaded cells for each technique throughout the whole range of activity classes.

Table 6. Retrieval results of top 1\% for MDDR-DS1 dataset for (ECFC_4) descriptor.

\begin{tabular}{cccccc}
\hline DS1 & \multicolumn{3}{c}{ Previous Studies } & & Proposed Methods \\
Retrieval Result 1\% & & & & & \\
Activity Index & TAN & BIN & SQB & SDBN & MLP \\
\hline 31420 & 69.69 & 74.08 & 73.73 & 74.21 & 82.1416 \\
71523 & 25.94 & 28.26 & 26.84 & 27.97 & 49.4118 \\
37110 & 9.63 & 26.05 & 24.73 & 26.03 & 45.5639 \\
\hline
\end{tabular}


Table 6. Cont.

\begin{tabular}{cccccc}
\hline DS1 & \multicolumn{3}{c}{ Previous Studies } & \multicolumn{2}{c}{ Proposed Methods } \\
$\begin{array}{c}\text { Retrieval Result 1\% } \\
\text { Activity Index }\end{array}$ & TAN & BIN & SQB & SDBN & MLP \\
\hline 31432 & 35.82 & 39.23 & 36.66 & 39.79 & 45.5957 \\
42731 & 17.77 & 21.68 & 21.17 & 23.06 & 32.0546 \\
6233 & 13.87 & 14.06 & 12.49 & 19.29 & 22.9708 \\
6245 & 6.51 & 6.31 & 6.03 & 6.27 & 5.36313 \\
7701 & 8.63 & 11.45 & 11.35 & 14.05 & 12.0918 \\
6235 & 9.71 & 10.84 & 10.15 & 12.87 & 10.7767 \\
78374 & 13.69 & 14.25 & 13.08 & 17.47 & 21.9196 \\
78331 & 7.17 & 6.03 & 5.92 & 9.93 & 9.70199 \\
\hline Mean & 19.86 & 22.93 & 22.01 & 24.63091 & 30.69 \\
\hline Shaded cells & 1 & 0 & 0 & 3 & 7 \\
\hline
\end{tabular}

Table 7. Retrieval results of top 5\% for MDDR-DS1 dataset for (ECFC_4) descriptor.

\begin{tabular}{cccccc}
\hline $\begin{array}{c}\text { DS1 } \\
\text { Retrieval Result 5\% }\end{array}$ & \multicolumn{3}{c}{ Previous Studies } & & Proposed Methods \\
Activity Index & TAN & BIN & SQB & SDBN & MLP \\
\hline 31420 & 83.49 & 87.61 & 87.22 & 89.03 & 87.3628 \\
71523 & 48.92 & 52.72 & 48.70 & 65.17 & 75.8289 \\
37110 & 21.01 & 48.20 & 45.62 & 41.25 & 71.4536 \\
31432 & 74.29 & 77.57 & 70.44 & 79.87 & 84.1489 \\
42731 & 29.68 & 26.63 & 24.35 & 31.92 & 51.3644 \\
6233 & 27.68 & 23.49 & 20.04 & 29.31 & 49.443 \\
6245 & 16.54 & 14.86 & 13.72 & 21.06 & 16.0894 \\
7701 & 24.09 & 27.79 & 26.73 & 28.43 & 29.7449 \\
6235 & 20.06 & 23.78 & 22.81 & 27.82 & 28.7379 \\
78374 & 20.51 & 20.20 & 19.56 & 19.09 & 36.7857 \\
78331 & 16.20 & 11.80 & 11.37 & 16.21 & 24.1391 \\
\hline Mean & 34.77 & 37.70 & 35.51 & 40.83273 & 50.463 \\
\hline Shaded cells & 0 & 0 & 0 & 2 & 9 \\
\hline
\end{tabular}

Table 8. Top 1\% retrieval results for MDDR-DS2 dataset for descriptor (ECFC 4).

\begin{tabular}{cccccc}
\hline $\begin{array}{c}\text { DS2 } \\
\text { Retrieval Result 1\% } \\
\text { Activity Index }\end{array}$ & TAN & BIN & SQB & SDBN & MLP \\
\hline 7707 & 61.84 & 72.18 & 72.09 & 83.19 & 86.4706 \\
7708 & 47.03 & 96.00 & 95.68 & 94.82 & 97.3077 \\
31420 & 65.10 & 79.82 & 78.56 & 79.27 & 71.7699 \\
42710 & 81.27 & 76.27 & 76.82 & 74.81 & 82.9091 \\
64100 & 80.31 & 88.43 & 87.80 & 93.65 & 94.2769 \\
64200 & 53.84 & 70.18 & 70.18 & 71.16 & 35.5696 \\
64220 & 38.64 & 68.32 & 67.58 & 68.71 & 88.5333 \\
64500 & 30.56 & 81.20 & 79.20 & 75.62 & 62.8571 \\
64350 & 80.18 & 81.89 & 81.68 & 85.21 & 91.8557 \\
75755 & 87.56 & 98.06 & 98.02 & 96.52 & 90.5727 \\
\hline Mean & 62.63 & 81.24 & 80.76 & 82.296 & 80.21226 \\
\hline Shaded cells & 0 & 3 & 0 & 1 & 6 \\
\hline
\end{tabular}


Table 9. Top 5\% retrieval results for MDDR-DS2 dataset for descriptor (ECFC 4).

\begin{tabular}{cccccc}
\hline DS2 & \multicolumn{3}{c}{ Previous Studies } & & Proposed Methods \\
Retrieval Result 5\% & & & & SQ \\
Activity Index & TAN & BIN & SQB & SDBN & MLP \\
\hline 7707 & 70.39 & 74.81 & 74.37 & 73.9 & 94.2157 \\
7708 & 56.58 & 99.61 & 99.61 & 98.22 & 98.7179 \\
31420 & 88.19 & 95.46 & 94.88 & 95.64 & 92.9381 \\
42710 & 88.09 & 92.55 & 91.09 & 90.12 & 88 \\
64100 & 93.75 & 99.22 & 99.03 & 99.05 & 96.6615 \\
64200 & 77.68 & 99.2 & 99.38 & 93.76 & 38.6076 \\
64220 & 52.19 & 91.32 & 90.62 & 96.01 & 93.2381 \\
64500 & 44.8 & 94.96 & 92.48 & 91.51 & 71.2698 \\
64350 & 91.71 & 91.47 & 90.78 & 86.94 & 95.3608 \\
75755 & 94.82 & 98.35 & 98.37 & 91.6 & 93.8767 \\
\hline Mean & 75.82 & 93.70 & 93.06 & 91.675 & 86.28862 \\
\hline Shaded cells & 0 & 4 & 3 & 2 & 2 \\
\hline
\end{tabular}

Table 10. Top 1\% retrieval results for MDDR-DS3 dataset for descriptor (ECFC 4).

\begin{tabular}{|c|c|c|c|c|c|}
\hline \multirow{2}{*}{$\begin{array}{c}\text { Ds3 } \\
\text { Retrieval Result 1\% } \\
\text { Activity Index }\end{array}$} & \multicolumn{4}{|c|}{ Previous Studies } & \multirow{2}{*}{$\begin{array}{c}\text { Proposed Methods } \\
\text { MLP } \\
\end{array}$} \\
\hline & TAN & BIN & SQB & SDBN & \\
\hline 9249 & 12.12 & 15.33 & 10.99 & 19.47 & 39.7556 \\
\hline 12455 & 6.57 & 9.37 & 7.03 & 13.29 & 9.8 \\
\hline 12464 & 8.17 & 8.45 & 6.92 & 12.91 & 31.84 \\
\hline 31281 & 16.95 & 18.29 & 18.67 & 23.62 & 61.8 \\
\hline 43210 & 6.27 & 7.34 & 6.83 & 14.23 & 17.5789 \\
\hline 71522 & 3.75 & 4.08 & 6.57 & 11.92 & 6.42857 \\
\hline 75721 & 17.32 & 20.41 & 20.38 & 29.08 & 57.5667 \\
\hline 78331 & 6.31 & 7.51 & 6.16 & 11.93 & 41.3 \\
\hline 78348 & 10.15 & 9.79 & 8.99 & 9.17 & 12.2 \\
\hline 78351 & 9.84 & 13.68 & 12.5 & 18.13 & 14.3024 \\
\hline Mean & 9.75 & 11.43 & 10.50 & 16.375 & 29.257217 \\
\hline Shaded cells & 0 & 0 & 0 & 3 & 7 \\
\hline
\end{tabular}

Table 11. Top 5\% retrieval results for MDDR-DS3 dataset for descriptor (ECFC 4).

\begin{tabular}{cccccc}
\hline $\begin{array}{c}\text { Ds3 Retrieval Result 5\% } \\
\text { Activity Index }\end{array}$ & TAN & BIN & SQB & SDBN & MLP \\
\hline 9249 & 24.17 & 25.72 & 17.8 & 31.61 & 61.1556 \\
12455 & 10.29 & 14.65 & 11.42 & 16.29 & 27.1429 \\
12464 & 15.22 & 16.55 & 16.79 & 20.9 & 53.72 \\
31281 & 29.62 & 28.29 & 29.05 & 36.13 & 75.8 \\
43210 & 16.07 & 14.41 & 14.12 & 22.09 & 36.2105 \\
71522 & 12.37 & 8.44 & 13.82 & 14.68 & 15.9143 \\
75721 & 25.21 & 30.02 & 30.61 & 41.07 & 78.2333 \\
78331 & 15.01 & 12.03 & 11.97 & 17.13 & 78.2 \\
78348 & 24.67 & 20.76 & 21.14 & 26.93 & 25.4667 \\
78351 & 11.71 & 12.94 & 13.3 & 17.87 & 34.2667 \\
\hline Mean & 18.43 & 18.38 & 18.00 & 24.47 & 48.611 \\
\hline Shaded cells & 0 & 0 & 0 & 1 & 9 \\
\hline
\end{tabular}


Table 12. Top $1 \%$ retrieval results for MUV dataset for descriptor (ECFC 4).

\begin{tabular}{ccccc}
\hline $\begin{array}{c}\text { MUV Retrieval Result 1\% } \\
\text { Activity Index }\end{array}$ & TAN & $\begin{array}{c}\text { Previous Studies } \\
\text { BIN }\end{array}$ & SQB & $\begin{array}{c}\text { Proposed Method } \\
\text { MLP }\end{array}$ \\
\hline 466 & 3.1 & 6.33 & 1.38 & 6.66667 \\
548 & 8.62 & 14.89 & 11.38 & 28.6667 \\
600 & 3.79 & 6.33 & 5.52 & 14.6667 \\
644 & 7.59 & 11 & 8.97 & 14.6667 \\
652 & 2.76 & 7 & 3.79 & 12 \\
689 & 3.79 & 7.33 & 4.48 & 8 \\
692 & 0.69 & 5.33 & 1.38 & 6.66667 \\
712 & 4.14 & 8.22 & 5.17 & 8.66667 \\
713 & 3.1 & 5.89 & 2.76 & 6 \\
733 & 3.45 & 6.67 & 4.14 & 6 \\
737 & 2.41 & 5.11 & 1.72 & 7.33333 \\
810 & 2.07 & 6.78 & 1.72 & 6.66667 \\
832 & 6.55 & 12.55 & 8.28 & 16.6667 \\
846 & 9.66 & 13.11 & 12.41 & 16 \\
852 & 12.41 & 13.78 & 9.66 & 18 \\
858 & 1.72 & 5.11 & 1.38 & 7.33333 \\
859 & 1.38 & 4.89 & 2.41 & 6.66667 \\
\hline Mean & 4.542941 & 8.254118 & 5.091176 & 11.21569471 \\
\hline Shaded cells & 0 & 2 & 0 & 12 \\
\hline & & & & \\
\hline
\end{tabular}

Table 13. Top 5\% retrieval results for MUV dataset for descriptor (ECFC 4).

\begin{tabular}{ccccc}
\hline $\begin{array}{c}\text { MUV Retrieval Result 5\% } \\
\text { Activity Index }\end{array}$ & TAN & $\begin{array}{c}\text { Previous Studies } \\
\text { BIN }\end{array}$ & SQB & Proposed Method \\
MLP
\end{tabular}


Table 14. Top 1\% retrieval results for DUD dataset.

\begin{tabular}{|c|c|c|c|}
\hline \multirow{2}{*}{$\begin{array}{c}\text { DUD Retrieval Result 1\% } \\
\text { Activity Index }\end{array}$} & \multicolumn{2}{|c|}{ Previous Studies } & \multirow{2}{*}{$\begin{array}{c}\text { Proposed Method } \\
\text { MLP }\end{array}$} \\
\hline & TAN & SQB3 & \\
\hline FGFR1T & 2.5 & 2.92 & 3.17 \\
\hline FXA & 1.92 & 3.36 & 1.64 \\
\hline GART & 7.75 & 5.75 & 8.00 \\
\hline GBP & 13.27 & 15.96 & 3.46 \\
\hline GR & 2.31 & 3.21 & 3.08 \\
\hline HIVPR & 3.55 & 3.55 & 5.16 \\
\hline HIVRT & 1.63 & 1.86 & 5.00 \\
\hline HMGA & 6.29 & 5.43 & 11.67 \\
\hline HSP90 & 1.62 & 4.05 & 4.21 \\
\hline MR & 5.33 & 5.33 & 10.00 \\
\hline NA & 2.24 & 5.31 & 5.20 \\
\hline PR & 1.85 & 2.22 & 4.29 \\
\hline Mean & 4.19 & 4.91 & 5.41 \\
\hline Shaded cells & 0 & 4 & 8 \\
\hline
\end{tabular}

Table 15. Top 5\% retrieval results for DUD dataset.

\begin{tabular}{|c|c|c|c|}
\hline \multirow{2}{*}{$\begin{array}{c}\text { DUD Retrieval Result 5\% } \\
\text { Activity Index }\end{array}$} & \multicolumn{2}{|c|}{ Previous Studies } & \multirow{2}{*}{$\begin{array}{c}\text { Proposed Method } \\
\text { MLP }\end{array}$} \\
\hline & TAN & SQB3 & \\
\hline FGFR1T & 6.67 & 7 & 8.17 \\
\hline FXA & 7.88 & 8.29 & 7.95 \\
\hline GART & 22.25 & 23.25 & 25.00 \\
\hline GBP & 20.96 & 30.96 & 10.00 \\
\hline GR & 6.41 & 8.46 & 7.69 \\
\hline HIVPR & 11.77 & 11.29 & 13.87 \\
\hline HIVRT & 4.88 & 6.98 & 9.09 \\
\hline HMGA & 10.29 & 13.14 & 21.11 \\
\hline HSP90 & 8.11 & 8.38 & 13.68 \\
\hline MR & 9.33 & 10 & 16.25 \\
\hline NA & 5.1 & 9.8 & 10.00 \\
\hline PR & 4.81 & 5.19 & 7.14 \\
\hline Mean & 9.87 & 11.90 & 12.50 \\
\hline Shaded cells & 0 & 3 & 9 \\
\hline
\end{tabular}

When comparing the MDDR-DS1 recall results for the top 1\% and 5\% in Tables 6 and 7 , the suggested enhanced Siamese MLP technique was clearly superior to the benchmark TAN method and prior studies BIN, SQB, and SDBN in terms of the mean and number of shaded cells. The suggested technique has the highest mean value (30.69) in Table 6, followed by SDBN, BIN, SQB, and lastly, TAN methods. In the suggested approach, the shaded cells have a value of (7). The suggested approach has the highest mean value (50.463) in Table 7, followed by SDBN, BIN, SQB, and lastly TAN methods. In the suggested technique, shaded cells have a value of (9).

Furthermore, the MDDR-DS2 recall values obtained at the top 1\%, as shown in Table 8 , demonstrate that the suggested Siamese MLP technique outperforms the benchmark TAN method. In view of the number of shaded cells, the MLP approach produced the best retrieval recall results, and the suggested method's mean value is extremely close to that of prior research. However, by comparison, The MDDR-DS2 recall values obtained at the top $5 \%$ in Table 9 clearly shows that the suggested Siamese MLP approach outperforms the benchmark TAN method only. In terms of the mean and number of shaded cells, the BIN 
approach produced the best retrieval recall results. Next, the second is SQB, SDBN, and finally, TAN in view of the mean value.

In addition, the MDDR-DS3 recall values recorded at the top 1\% and 5\% in Tables 10 and 11, respectively, show that the proposed enhanced Siamese MLP method is obviously superior to the benchmark TAN method and methods from other studies. Likewise, in Table 10, the proposed method gave the best retrieval recall results in view of the mean and number of shaded cells, compared to prior studies and benchmark TAN, followed by SDBN, BIN, SQB, and finally, TAN methods. By comparison, in Table 11, the suggested enhanced Siamese MLP method was obviously superior to the benchmark TAN method and other studies. The second one is SDBN, followed by TAN, BIN, and SQB.

Moreover, the MUV dataset recall values recorded at the top 1\% and 5\% in Tables 12 and 13, respectively, show that the proposed enhanced Siamese MLP method is obviously superior to the benchmark TAN method and other studies. Likewise, in Table 12, the proposed method gave the best retrieval recall results in view of the mean and number of shaded cells, compared to the TAN method and methods from other studies, followed by BIN, SQB, and finally, TAN methods. However, by comparison, in Table 13, the proposed enhanced Siamese MLP method was obviously superior to the benchmark TAN method and methods from other studies. Next, the second one is BIN, followed by SQB and TAN.

Moreover, the DUD dataset recall values recorded at the top 1\% and 5\% in Tables 14 and 15, respectively, show that the proposed enhanced Siamese MLP method is obviously superior to the benchmark TAN method and methods from other studies. Likewise, in Table 14, the proposed method gave the best retrieval recall results in view of the mean and number of shaded cells, compared to the previous study and benchmark TAN. Furthermore, in Table 15, the proposed enhanced Siamese MLP method was obviously superior to the benchmark TAN method and the previous study SQB.

The experimental results for pruning nodes on MDDR-DS1, MDDR-DS2, MDDR-DS3, MUV, and DUD datasets are shown in Figures 4-13, respectively. In these figures, the $x$ axis represents the pruning ratio starting from $0 \%$ and ending with $90 \%, 95 \%, 95 \%, 70 \%$, and $98 \%$ in DS1, DS2, DS3, MUV, and DUD datasets, respectively. They y axis represents the level of retrieval recall values for each class in the dataset. The classes of molecules are represented as color lines. The tables that contain on pruning ratio of recall values for each dataset are available as Supplementary Materials.

Figure 4 shows the level of the retrieval recall values at different pruning ratios for each class at the top 1\% in DS1. We note that the recall values of most classes remain the same until they reach $80 \%$ of the pruning ratio, while some classes increased slightly, such as class 7 , and decreased by a little in others, such as classes 2 and 9. Furthermore, Figure 5 shows the level of retrieval recall values at different pruning ratios for each class at the top $5 \%$ in DS1. The recall values of most classes remain the same values until they reach $80 \%$ of the pruning ratio, while some classes decreased, such as classes 2 and 10.

Figure 6 shows the level of retrieval recall values at different pruning ratios for each class at the top $1 \%$ in DS2. We note that the recall values of most classes remain the same until they reach $90 \%$ of the pruning ratio, while some classes increased slightly, such as classes 5 and 8 , and decreased by a little in others, such as classes 1,3, and 4 . Furthermore, Figure 7 shows the level of retrieval recall values at different pruning ratios for each class at the top 5\% in DS2. The recall values of most classes remain the same until they reach $90 \%$ of the pruning ratio, except for class 6 , which remains until more than $95 \%$ of the pruning ratio, while some classes decreased by a little, such as class 4 , or increased slightly, such as class 5 .

Figure 8 shows the level of retrieval recall values at different pruning ratios for each class at the top $1 \%$ in DS3. We note that the recall values of most classes remain the same until they reach $80 \%$ of the pruning ratio, while some classes increased slightly, such as class 4 , a more than $95 \%$ pruning ratio, and decreased by a little in others, such as classes 1 , 7 , and 8 . Furthermore, Figure 9 shows the retrieval recall values at different pruning ratios for each class at the top 5\% in DS3. The recall values of most classes remain the same until 
they reach $80 \%$ of the pruning ratio, except for class 4 , which increased until more than $95 \%$, while some classes decreased by a little, such as classes $1,3,7$, and 8 .

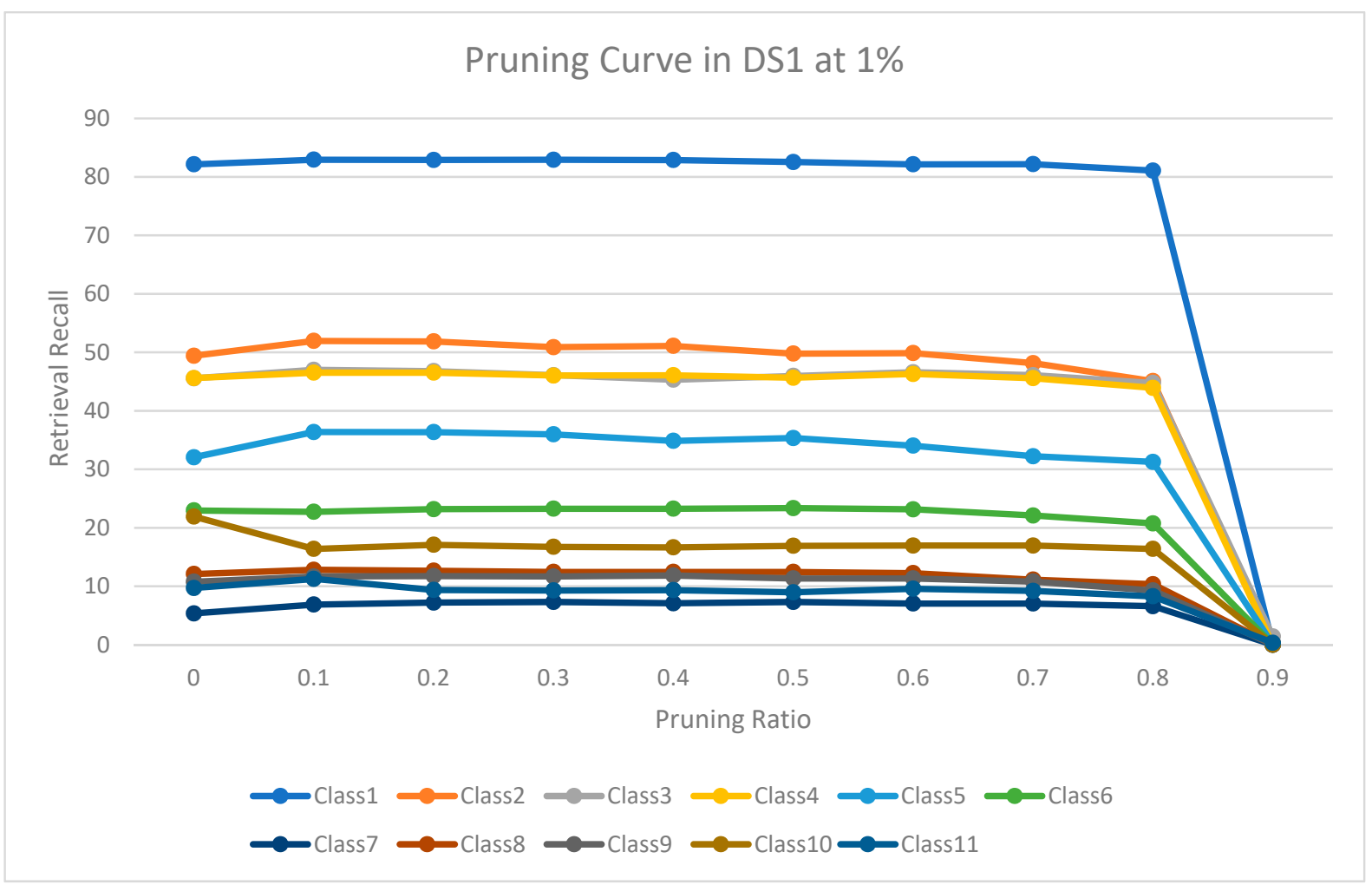

Figure 4. The level of retrieval recall values at different percentages of pruning at the top 1\% in MDDR-DR1.

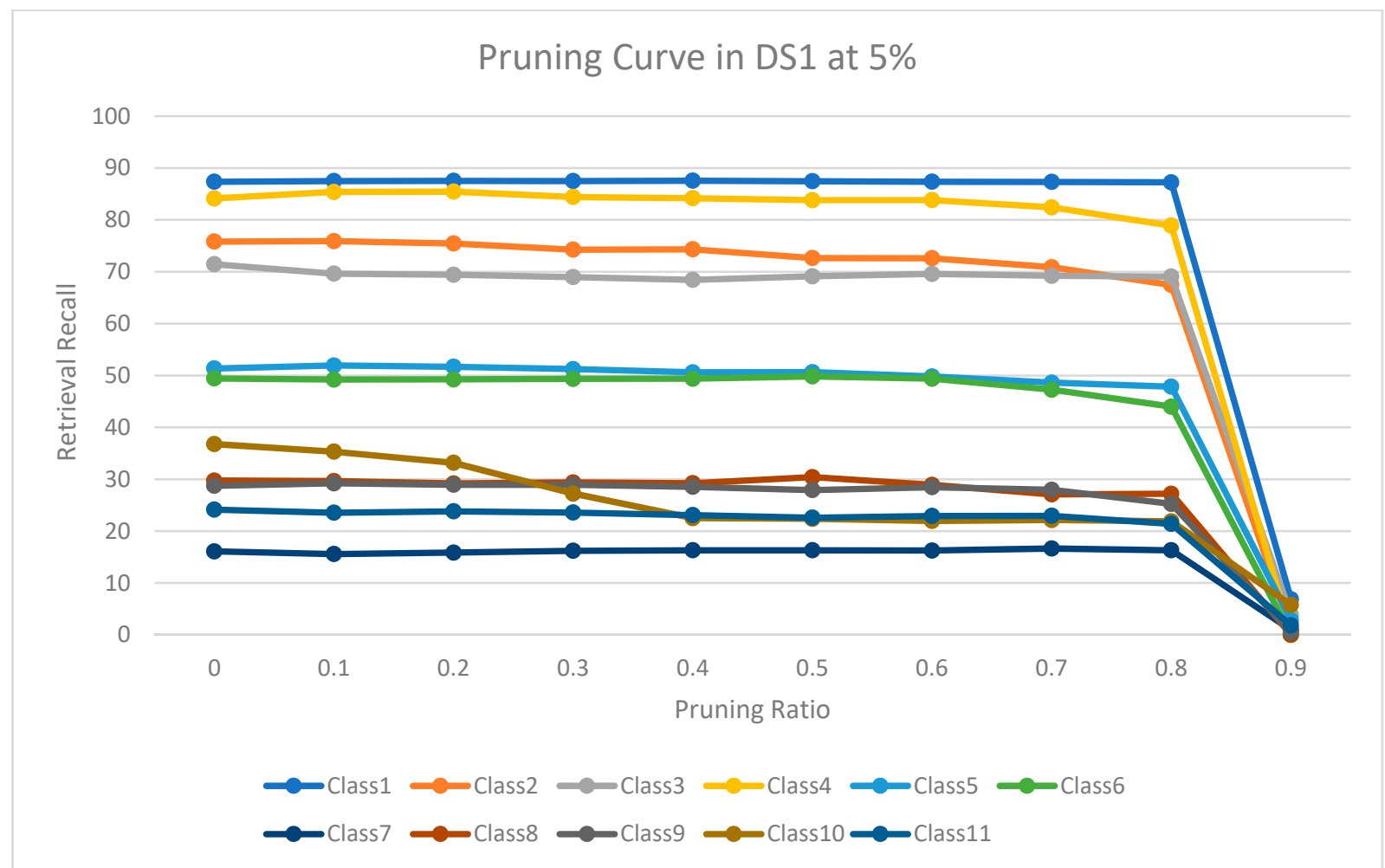

Figure 5. The level of retrieval recall values at different percentages of pruning at the top 5\% in MDDR-DR1. 


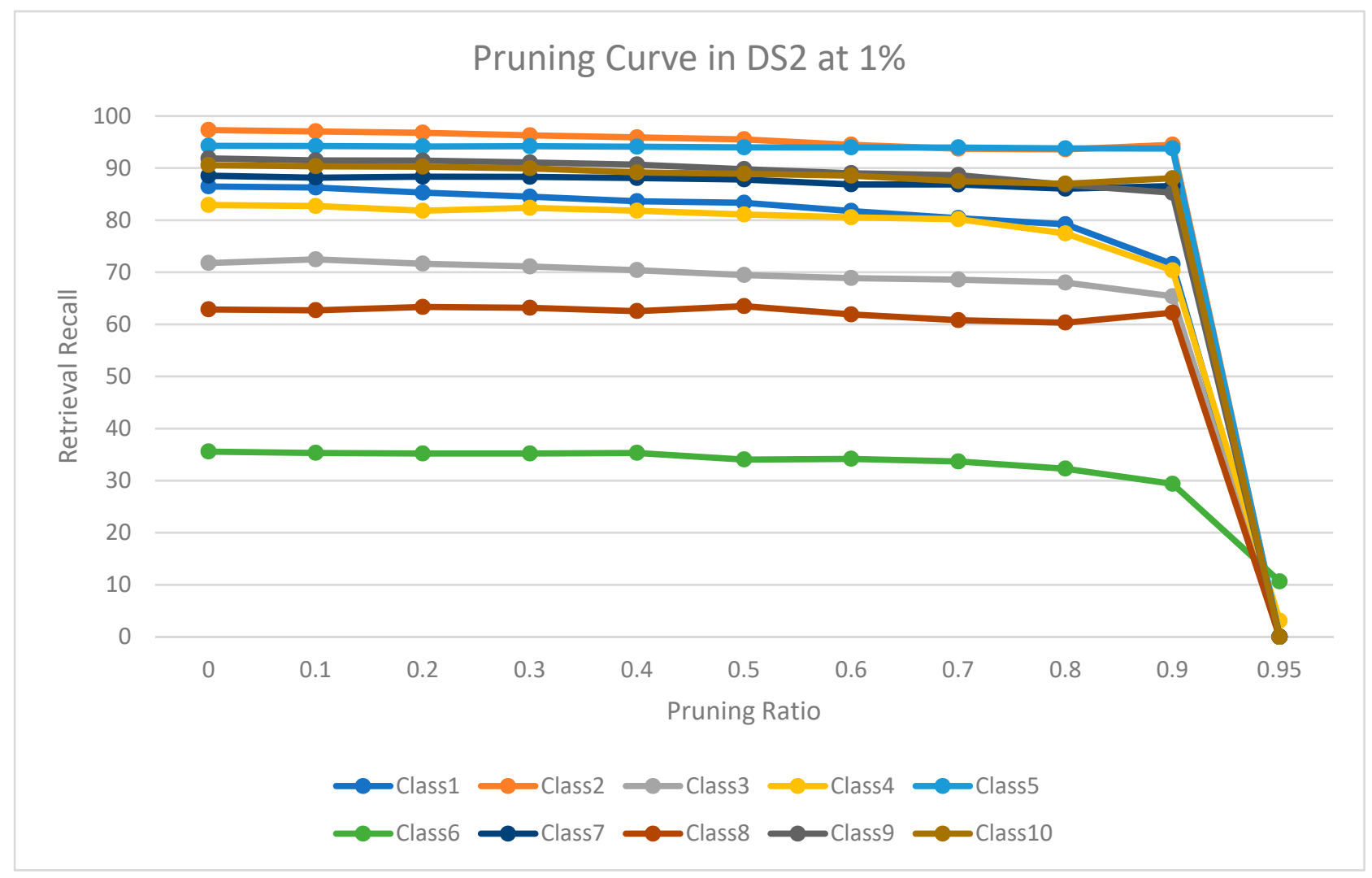

Figure 6. The level of retrieval recall values at different percentages of pruning at the top 1\% in DDR-DR2.

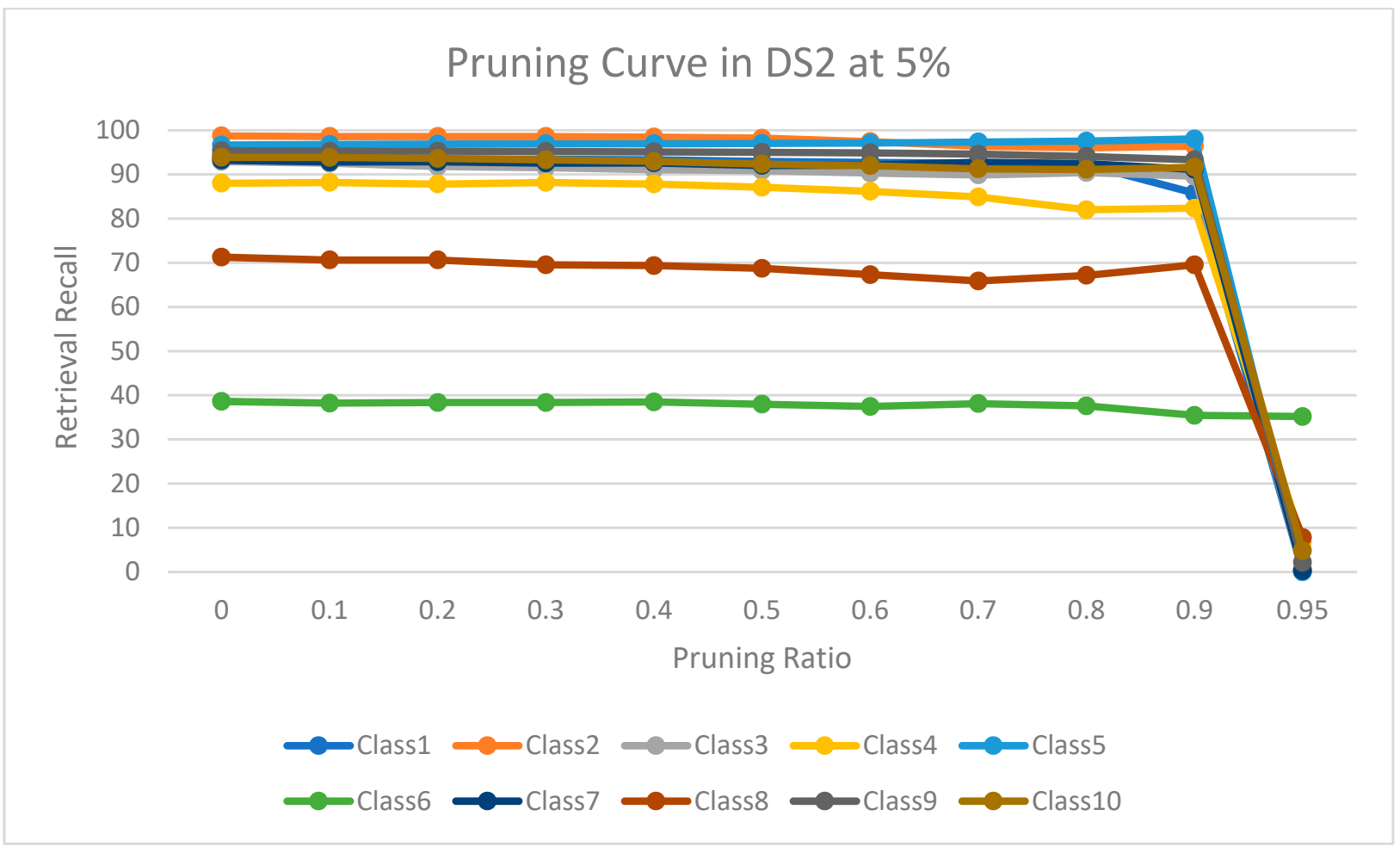

Figure 7. The level of retrieval recall values at different percentages of pruning at the top 5\% in MDDR-DR2. 


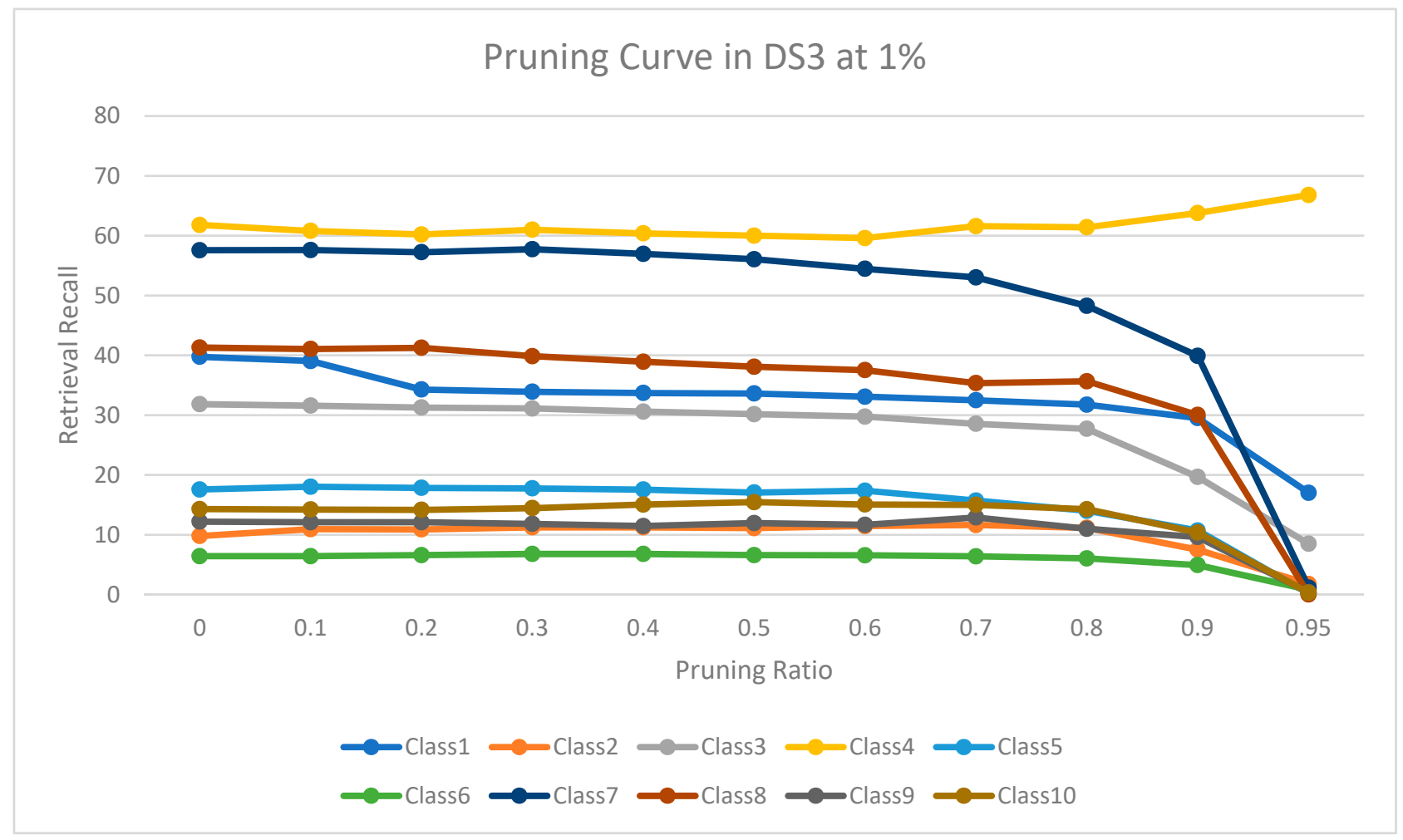

Figure 8. The level of retrieval recall values at different percentages of pruning at the top 1\% in DR3-MDDR.

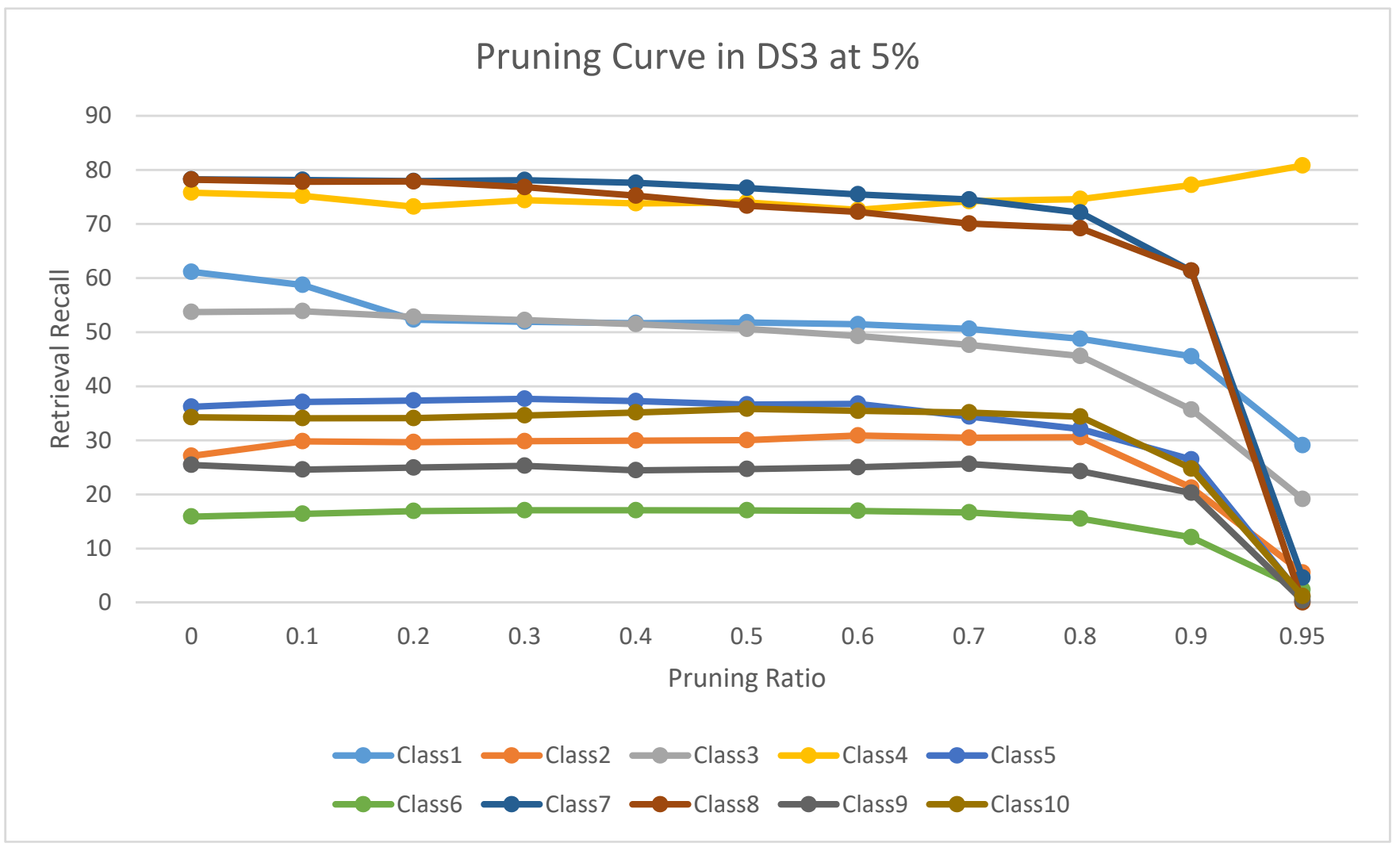

Figure 9. The level of retrieval recall values at different percentages of pruning at the top 5\% in DR3-MDDR. 


\section{Pruning Curve in MUV at $1 \%$}

25
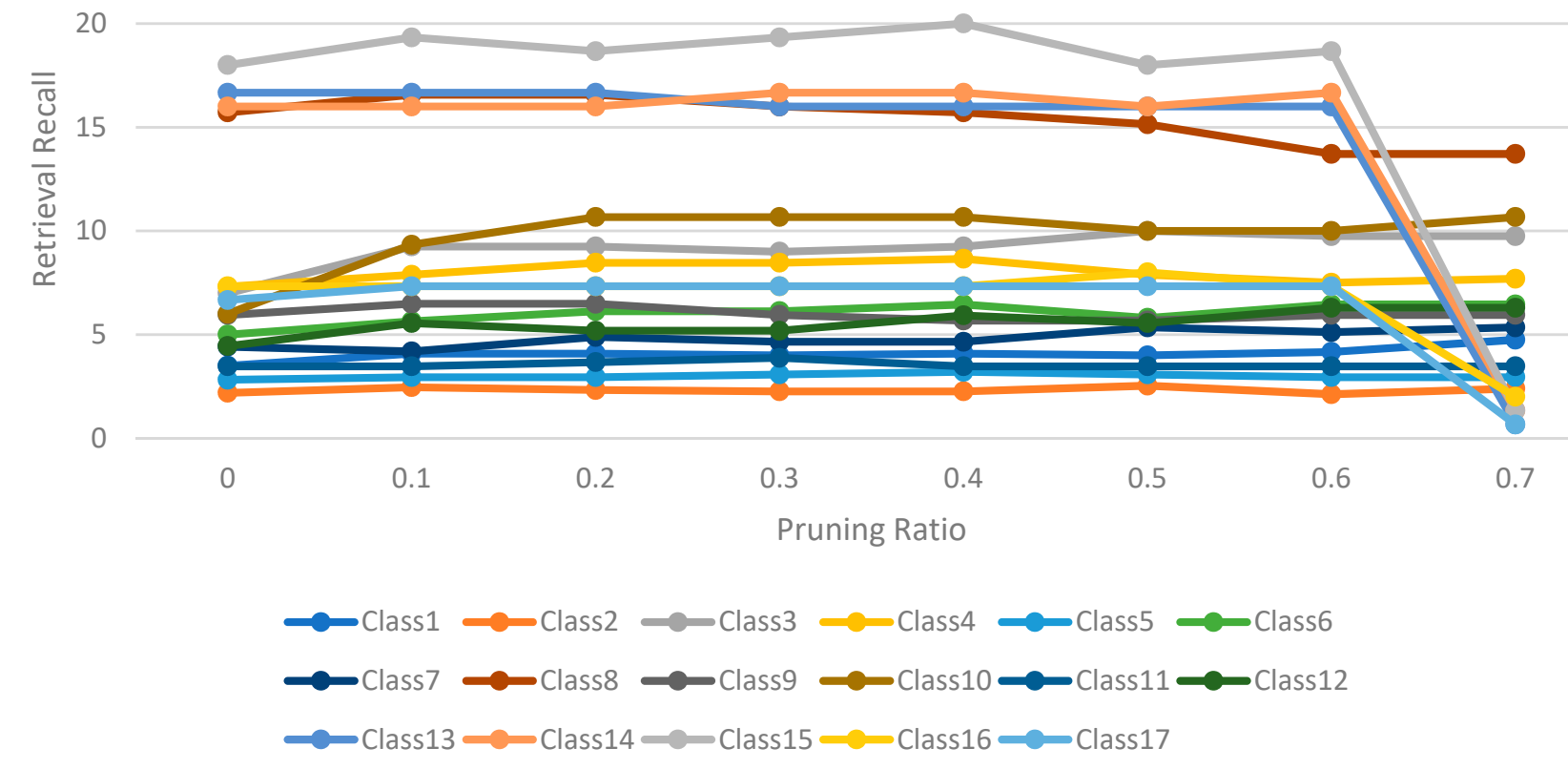

Figure 10. The level of retrieval recall values at different percentages of pruning at the top $1 \%$ in MUV dataset.

\section{Pruning Curve in MUV at 5\%}

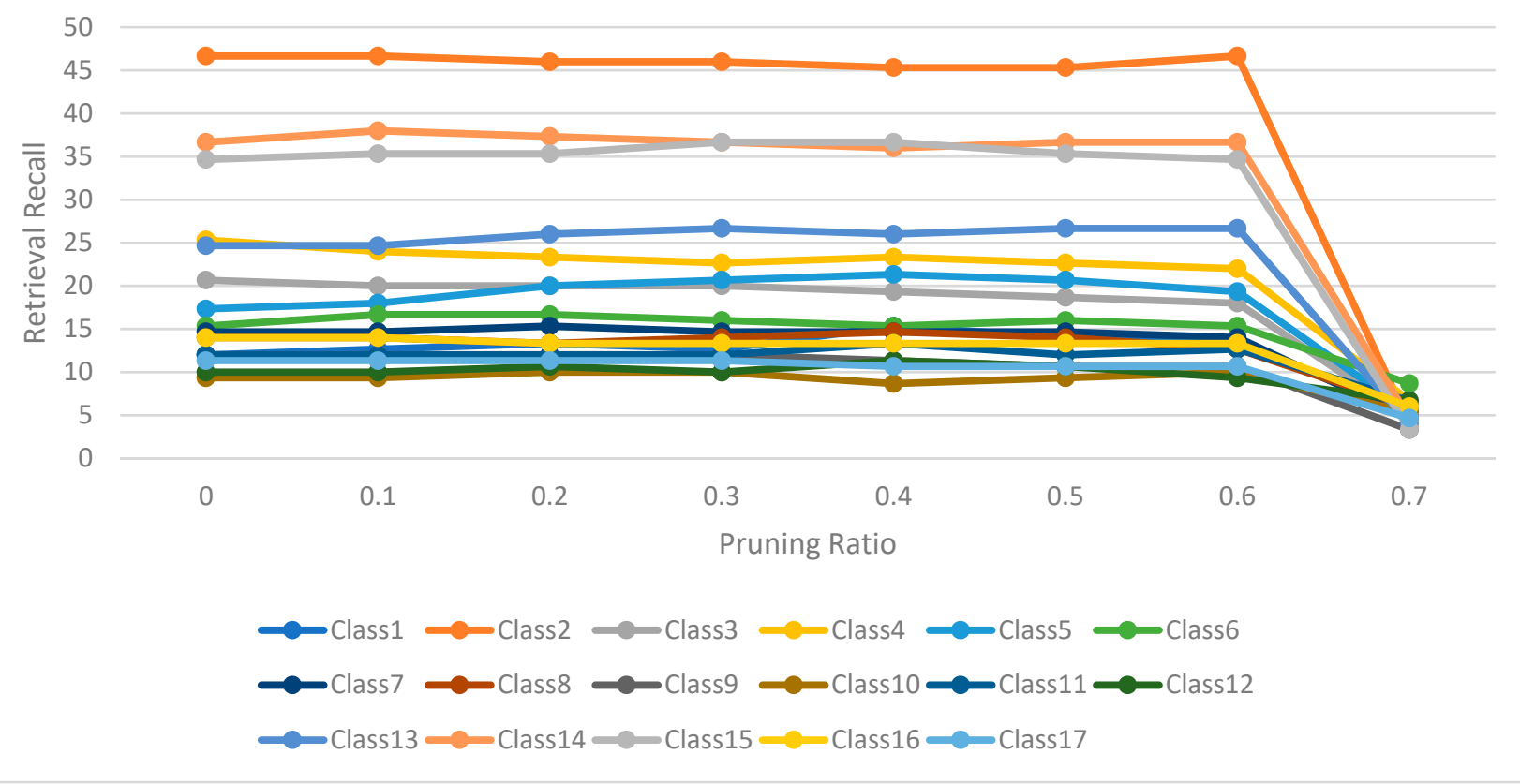

Figure 11. The level of retrieval recall values at different percentages of pruning at the top 5\% in MUV dataset. 


\section{Pruning Curve in DUD at $1 \%$}

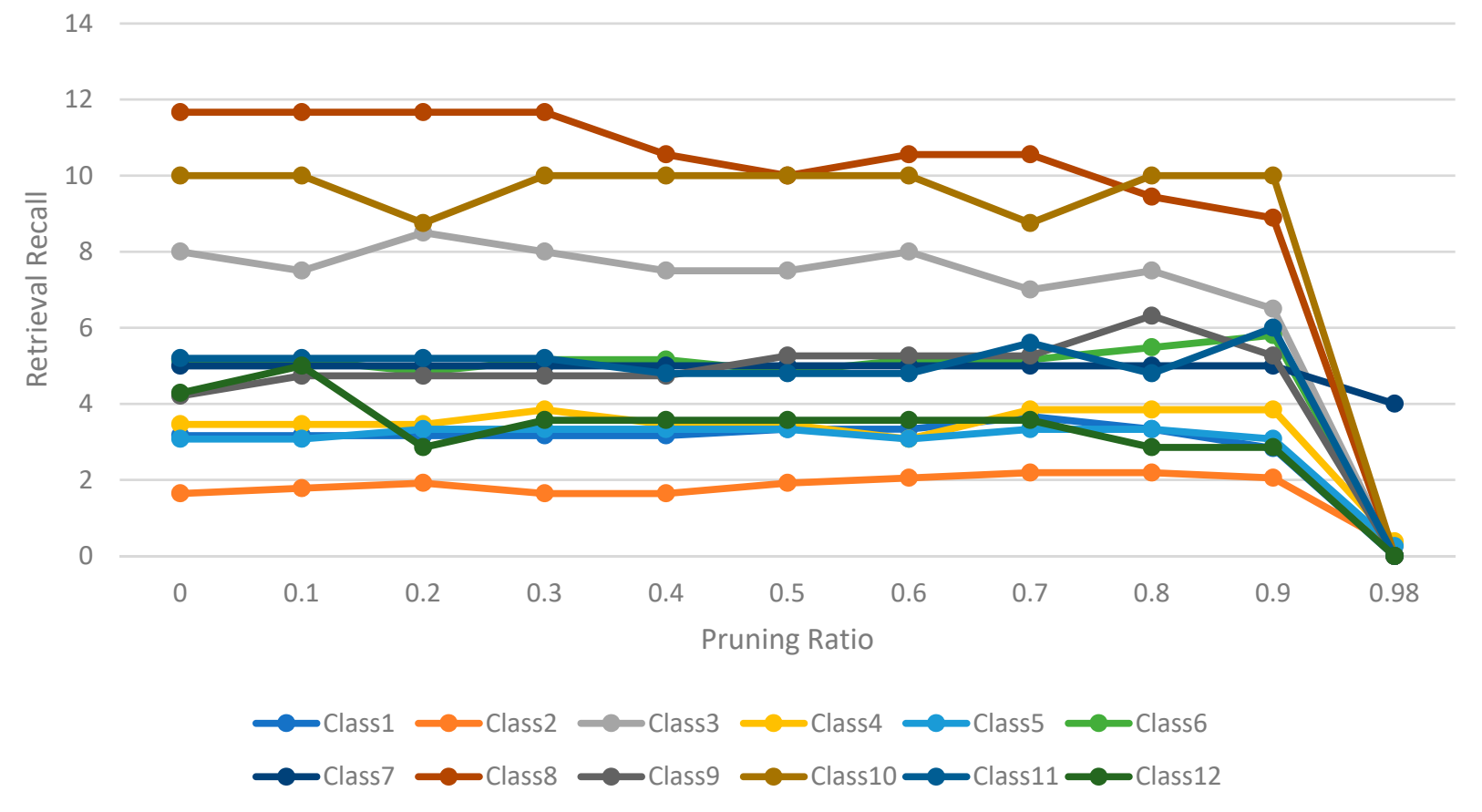

Figure 12. The level of retrieval recall values at different percentages of pruning at the top 1\% in DUD dataset.

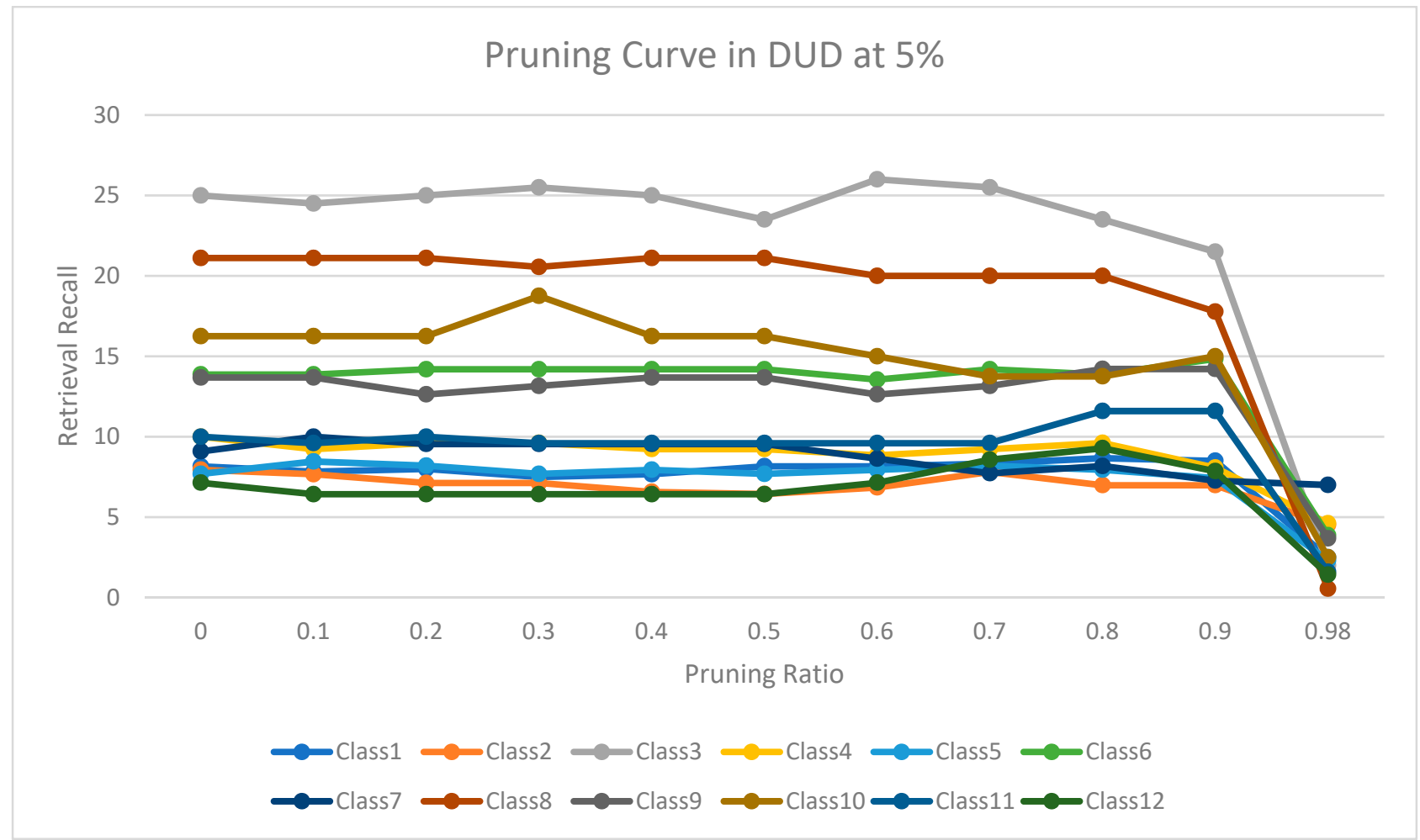

Figure 13. The level of retrieval recall values at different percentages of pruning at the top 5\% in DUD dataset.

Figure 4 shows the level of the retrieval recall values at different pruning ratios for each class at the top $1 \%$ in DS1. We note that the recall values of most classes remain the same until they reach $80 \%$ of the pruning ratio, while some classes increased slightly, such 
as class 7, and decreased by a little in others, such as classes 2 and 9 . Furthermore, Figure 5 shows the level of retrieval recall values at different pruning ratios for each class at the top $5 \%$ in DS1. The recall values of most classes remain the same values until they reach $80 \%$ of the pruning ratio, while some classes decreased, such as classes 2 and 10.

Figure 6 shows the level of retrieval recall values at different pruning ratios for each class at the top $1 \%$ in DS2. We note that the recall values of most classes remain the same until they reach $90 \%$ of the pruning ratio, while some classes increased slightly, such as classes 5 and 8 , and decreased by a little in others, such as classes 1, 3, and 4. Furthermore, Figure 7 shows the level of retrieval recall values at different pruning ratios for each class at the top 5\% in DS2. The recall values of most classes remain the same until they reach $90 \%$ of the pruning ratio, except for class 6 , which remains until more than $95 \%$ of the pruning ratio, while some classes decreased by a little, such as class 4, or increased slightly, such as class 5 .

Figure 8 shows the level of retrieval recall values at different pruning ratios for each class at the top $1 \%$ in DS3. We note that the recall values of most classes remain the same until they reach $80 \%$ of the pruning ratio, while some classes increased slightly, such as class 4 , a more than $95 \%$ pruning ratio, and decreased by a little in others, such as classes 1 , 7 , and 8 . Furthermore, Figure 9 shows the retrieval recall values at different pruning ratios for each class at the top $5 \%$ in DS3. The recall values of most classes remain the same until they reach $80 \%$ of the pruning ratio, except for class 4 , which increased until more than 95\%, while some classes decreased by a little, such as classes 1, 3, 7, and 8.

Figure 10 shows the level of retrieval recall values at different pruning ratios for each class at the top $1 \%$ in MUV. We note that the recall values of most classes remained the same until they reached $60 \%$ of the pruning ratio, while some classes increased slightly, such as classes 3 and 10, and decreased by a little in others, such as class 8 . Moreover, Figure 11 shows the retrieval recall values at different pruning ratios for each class at the top 5\% in MUV. The recall values of most classes remained the same until they reached $60 \%$ of the pruning ratio, while some classes increased slightly, such as classes 1,3 and 5, and decreased by a little in others, such as classes 3 and 4 .

Furthermore, Figure 12 shows the level of retrieval recall values at different pruning ratios for each class at the top 1\% in DUD. We note that the recall values of most classes remained the same until they reached $80 \%$ of the pruning ratio, while some classes increased slightly, such as classes 4,6, and 9, and decreased by a little in others, such as classes 3, 8, and 12. Moreover, Figure 13 shows the retrieval recall values at different pruning ratios for each class at the top 5\% in DUD. The recall values of most classes remained the same until they reached $80 \%$ of the pruning ratio, while some classes increased slightly, such as classes 11 and 12, and decreased by a little in others, such as classes 3 and 10.

Moreover, the Kendall W concordance test has been used; Table 16 shows the ranking of the enhanced Siamese multilayer perceptron method based on previous studies of TAN, BIN, SQB, and SDBN using Kendall W test results for MDDR-DS1, MDDR-DS2, MDDRDS3, MUV, and DUD at the top $1 \%$ and $5 \%$. The first method is the benchmark method, which is the Tanimoto coefficient TAN; the second method is Bayesian inference [24]; the third method is quantum similarity search SQB-Complex [28]; the last method is multidescriptor-based on Stack of deep belief networks SDBN [31]. The results of the Kendall W test of the top $1 \%$ for all used datasets show that the values of associated probability $(p)$ are less than 0.05 . This indicates that the enhanced Siamese multilayer perceptron method is significant in the top $1 \%$ for all cases. As a result, the overall ranking of all methods indicates that the enhanced Siamese multilayer perceptron method is superior to previous studies and benchmark TAN. The overall ranking for methods showed that MLP has the top ranks among other methods. This is the same as with the results of the Kendall W test of the top 5\%; the results show that the values of associated probability (p) are less than 0.05 . This indicates that the enhanced Siamese multilayer perceptron method is significant in the top 5\%. As a result, the overall ranking of all methods indicates that the enhanced Siamese multilayer perceptron method is superior to previous studies for all datasets and 
the overall ranking for the method showed that Siamese multilayer perceptron method has the top ranks among other methods, except in DR2, in which the BIN method was better than MLP. Figures 14 and 15 show the ranking of the enhanced Siamese multilayer perceptron method based on TAN, BIN, SQB, and SDBN using the Kendall W test results for DS1, DS2,DS3, MUV, and DUD at the top $1 \%$ and $5 \%$.

Table 16. Ranking of enhanced Siamese multilayer perceptron method based on previous studies TAN, BIN, SQB, and SDBN using Kendall $\mathrm{W}$ test results.

\begin{tabular}{|c|c|c|c|c|c|}
\hline DataSet & Retrieval Percentage & $\mathbf{W}$ & $\mathbf{P}$ & \multicolumn{2}{|c|}{ Rank Methods } \\
\hline \multirow{10}{*}{ DS1 } & \multirow{5}{*}{$1 \%$} & \multirow{5}{*}{0.593} & \multirow{5}{*}{0.00003} & MLP & 4.27 \\
\hline & & & & SDBN & 4.00 \\
\hline & & & & $\mathrm{BIN}$ & 3.27 \\
\hline & & & & SQB & 1.73 \\
\hline & & & & TAN & 1.73 \\
\hline & \multirow{5}{*}{$5 \%$} & \multirow{5}{*}{0.588} & \multirow{5}{*}{0.000033} & MLP & 4.64 \\
\hline & & & & SDBN & 3.73 \\
\hline & & & & $\mathrm{BIN}$ & 2.82 \\
\hline & & & & TAN & 2.27 \\
\hline & & & & SQB & 1.55 \\
\hline \multirow{10}{*}{ DS2 } & \multirow{5}{*}{$1 \%$} & \multirow{5}{*}{0.3673} & \multirow{5}{*}{0.0053} & MLP & 3.70 \\
\hline & & & & BIN & 3.65 \\
\hline & & & & SDBN & 3.40 \\
\hline & & & & SQB & 2.85 \\
\hline & & & & TAN & 1.40 \\
\hline & \multirow{5}{*}{$5 \%$} & \multirow{5}{*}{0.34321} & \multirow{5}{*}{0.00821} & BIN & 4.15 \\
\hline & & & & SQB & 3.55 \\
\hline & & & & SDBN & 2.90 \\
\hline & & & & MLP & 2.70 \\
\hline & & & & TAN & 1.70 \\
\hline \multirow{10}{*}{ DS3 } & \multirow{5}{*}{$1 \%$} & \multirow{5}{*}{0.698} & \multirow{5}{*}{0.0000129} & MLP & 4.60 \\
\hline & & & & SDBN & 4.10 \\
\hline & & & & $\mathrm{BIN}$ & 2.80 \\
\hline & & & & SQB & 1.90 \\
\hline & & & & TAN & 1.60 \\
\hline & \multirow{5}{*}{$5 \%$} & \multirow{5}{*}{0.784} & \multirow{5}{*}{0.000002584} & MLP & 4.90 \\
\hline & & & & SDBN & 4.10 \\
\hline & & & & SQB & 2.10 \\
\hline & & & & TAN & 2.00 \\
\hline & & & & BIN & 1.90 \\
\hline
\end{tabular}


Table 16. Cont.

\begin{tabular}{|c|c|c|c|c|c|}
\hline DataSet & Retrieval Percentage & $\mathbf{W}$ & $\mathbf{P}$ & \multicolumn{2}{|c|}{ Rank Methods } \\
\hline \multirow{8}{*}{ MUV } & \multirow{4}{*}{$1 \%$} & \multirow{4}{*}{0.867} & \multirow{4}{*}{$1.35 \times 10^{-9}$} & MLP & 3.88 \\
\hline & & & & BIN & 3.12 \\
\hline & & & & SQB & 1.65 \\
\hline & & & & TAN & 1.35 \\
\hline & \multirow{4}{*}{$5 \%$} & \multirow{4}{*}{0.702} & \multirow{4}{*}{$8.24 \times 10^{-8}$} & MLP & 3.74 \\
\hline & & & & BIN & 3.03 \\
\hline & & & & SQB & 1.82 \\
\hline & & & & TAN & 1.41 \\
\hline \multirow{6}{*}{ DUD } & \multirow{3}{*}{$1 \%$} & \multirow{3}{*}{0.3115} & \multirow{3}{*}{$2.38 \times 10^{-2}$} & MLP & 2.50 \\
\hline & & & & SQB & 2.08 \\
\hline & & & & TAN & 1.42 \\
\hline & \multirow{3}{*}{$5 \%$} & \multirow{3}{*}{0.58333} & \multirow{3}{*}{0.0009118} & MLP & 2.67 \\
\hline & & & & SQB & 2.17 \\
\hline & & & & TAN & 1.17 \\
\hline
\end{tabular}

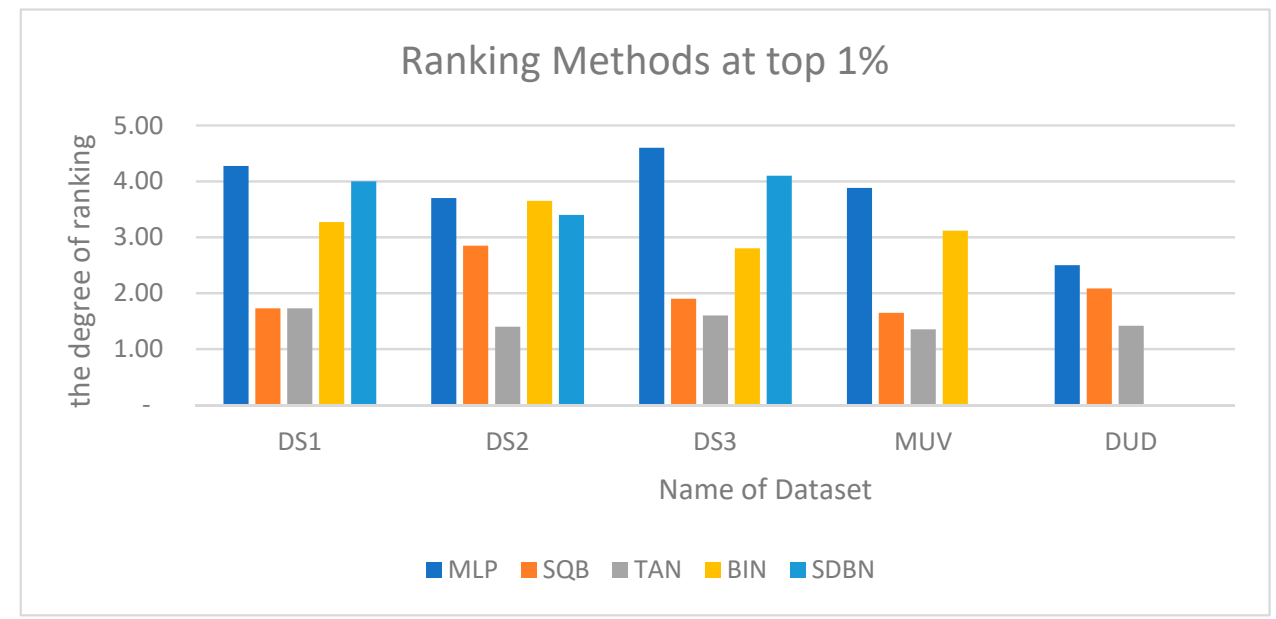

Figure 14. Ranking of enhanced Siamese multilayer perceptron method based on TAN, BIN, SQB, and SDBN using Kendall W test results for DS1, DS2, DS3, MUV, and DUD at top $1 \%$.

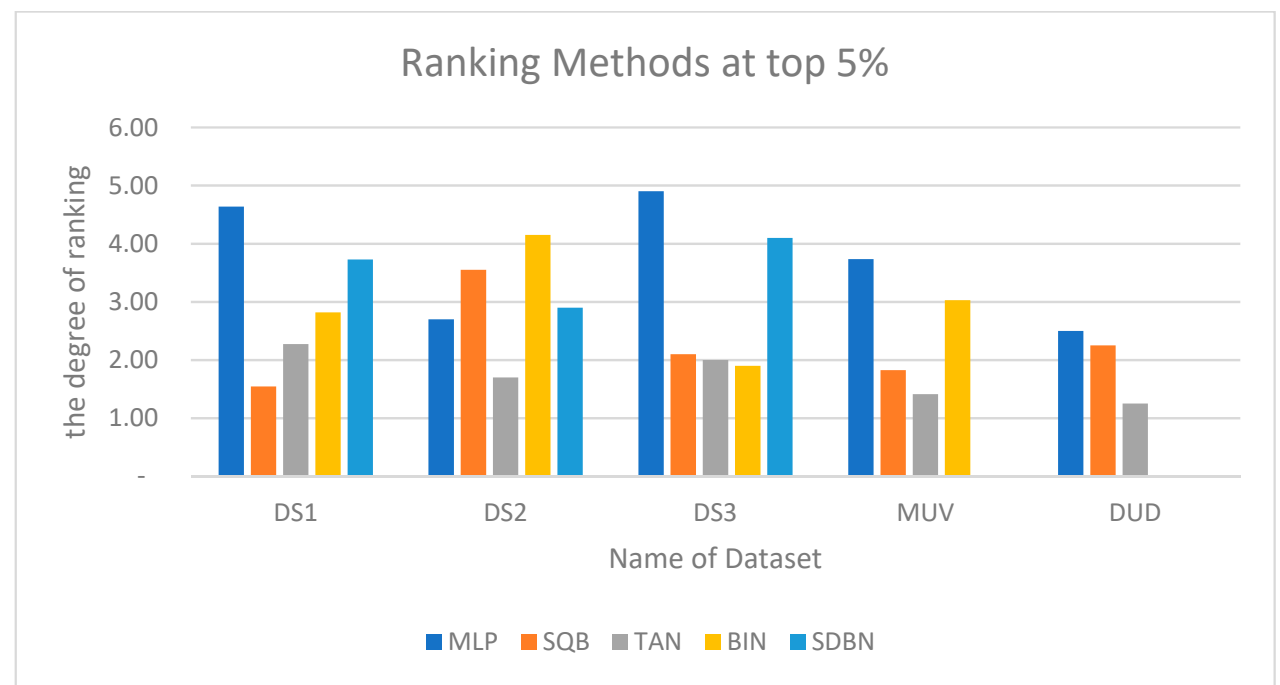

Figure 15. Ranking of enhanced Siamese multilayer perceptron method based on TAN, BIN, SQB, and SDBN using Kendall W test results for DS1, DS2, DS3, MUV, and DUD at top 5\%. 


\section{Conclusions}

Many techniques for capturing the biological similarity between a test compound and a known target ligand in LBVS have been established. LBVS is based on the premise that the target-binding behavior of related property compounds will be related. In spite of the good performances of the above methods compared to their prior, especially when dealing with molecules that have structurally homogenous active elements, the performances are not satisfied when dealing with molecules that are structurally heterogeneous. The main goal of this research was to improve the retrieval effectiveness of the similarity model, especially with molecules that are structurally heterogeneous. In this study, the Siamese multilayer perceptron similarity model has been enhanced by using two distance layers with a fuse layer that combines the results from two distance layers, and then multiple layers were added after the fusion layer, followed by pruning of the nodes that contribute less or nothing to the network during inference according to their signal-to-noise ratio. The results showed that the significance of the proposed method obviously outperformed the standard Tanimoto coefficient (TAN) and previous studies (BIN, SQB, and SDBN) at the top $1 \%$ and $5 \%$ for MDDR-DS1, MDDR-DS3, DUD, and MUV, which include heterogeneous classes. Additionally, the proposed method has the top rank for the top 1\% MDDR-DS2, which include homogeneous classes. Besides that, it is possible to reduce the number of nodes in the Siamese multilayer perceptron model while still keeping the effectiveness of recall on the same level when pruning $60 \%$ of nodes in MUV, $90 \%$ in DS2, and $80 \%$ in DS1, DS3, and DUD. Multiple molecular descriptors will be tested in this proposed method as future work.

Supplementary Materials: The following are available online, Support Information which contains Table S1: The structure-activity classes of the MUV dataset, Table S2: The MDDR-DS1 structure activity classes, Table S3: The MDDR-DS2 structure activity classes, Table S4: The MDDR-DS3 structure activity classes, Table S5: DUD structure activity classes. Also Experiment Results of proposed method in each query with pruning in excel file.

Author Contributions: Conceptualization, M.K.A. and N.S.; methodology, M.K.A. and N.S.; software, M.K.A.; validation, M.K.A. and N.S.; formal analysis, M.K.A. and N.S.; investigation, M.K.A. and N.S.; data curation, M.K.A.; writing—original draft, M.K.A.; writing—review and editing, M.K.A. and N.S.; supervision, N.S.; project administration, N.S.; funding acquisition, N.S. All authors have read and agreed to the published version of the manuscript.

Funding: This work was supported by the Ministry of Higher Education Project No: R.J130000.7828.4F 985, and Malaysia Ministry of Higher Education and Universiti Teknologi Malaysia Project No: Q.J130000.2551.21H38.

Institutional Review Board Statement: Not applicable.

Informed Consent Statement: Not applicable.

Data Availability Statement: The MDL Drug Data Report (MDDR) dataset is owned by www. accelrys.com (accessed on 31 October 2021). A license is required to access the data. Maximum unbiased validation (MUV) datasets are freely available at http:/ / www.pharmchem.tu-bs.de/lehre/ baumann/MUV.html (accessed on 31 October 2021). The DUD dataset is freely accessible online as a benchmarking set at http:/ / blaster.docking.org/dud/ (accessed on 31 October 2021). Software License: Python 3.7 in environment anaconda/Spyder was used with the following libraries: TensorFlow, Theano, Keras, Numpy, Pandas, and math. The license of statistics application (IBM spss) is licenseapp.utm.my.

Acknowledgments: I would like to thank the Islamic development bank (IsDB) for the scholarships. and Mosul University for encouraging me to continue with my studies.

Conflicts of Interest: The authors declare no conflict of interest.

Sample Availability: Not available. 


\section{References}

1. Hertzberg, R.P.; Pope, A.J. High-throughput screening: New technology for the 21st century. Curr. Opin. Chem. Biol. 2000, 4, 445-451. [CrossRef]

2. DiMasi, J.A.; Grabowski, H.G.; Hansen, R.W. Innovation in the pharmaceutical industry: New estimates of R\&D costs. J. Health Econ. 2016, 47, 20-33.

3. Carpenter, K.A.; Cohen, D.S.; Jarrell, J.T.; Huang, X. Deep learning and virtual drug screening. Future Med. Chem. 2018, 10, 2557-2567. [CrossRef]

4. Lavecchia, A.; Di Giovanni, C. Virtual screening strategies in drug discovery: A critical review. Curr. Med. Chem. 2013, 20, 2839-2860. [CrossRef]

5. Shoichet, B.K. Virtual screening of chemical libraries. Nature 2004, 432, 862-865. [CrossRef]

6. Cheng, T.; Li, Q.; Zhou, Z.; Wang, Y.; Bryant, S.H. Structure-based virtual screening for drug discovery: A problem-centric review. AAPS J. 2012, 14, 133-141. [CrossRef] [PubMed]

7. Chaudhary, K.K.; Mishra, N. A review on molecular docking: Novel tool for drug discovery. Databases $2016,4,3$.

8. Brown, N. Chemoinformatics—an introduction for computer scientists. ACM Comput. Surv. (CSUR) 2009, 41, 1-38. [CrossRef]

9. Cereto-Massagué, A.; Ojeda, M.J.; Valls, C.; Mulero, M.; Garcia-Vallvé, S.; Pujadas, G. Molecular fingerprint similarity search in virtual screening. Methods 2015, 71, 58-63. [CrossRef]

10. Willett, P. Similarity searching using 2D structural fingerprints. In Chemoinformatics and Computational Chemical Biology; Springer: Berlin/Heidelberg, Germany, 2010; pp. 133-158.

11. Fukunishi, Y. Structure-based drug screening and ligand-based drug screening with machine learning. Comb. Chem. High Throughput Screen. 2009, 12, 397-408. [CrossRef] [PubMed]

12. Narang, S.; Elsen, E.; Diamos, G.; Sengupta, S. Exploring sparsity in recurrent neural networks. arXiv 2017, arXiv:1704.05119.

13. Bromley, J.; Guyon, I.; Lecun, Y.; Säckinger, E.; Shah, R. Signature verification using a "Siamese" time delay neural network. Int. J. Pattern Recognit. Artif. Intel. 1993, 7, 669-688. [CrossRef]

14. Chicco, D. Siamese neural networks: An overview. Artif. Neural Netw. 2021, 2190, 73-94.

15. Blundell, C.; Cornebise, J.; Kavukcuoglu, K.; Wierstra, D. Weight uncertainty in neural networks. arXiv 2015, arXiv:1505.05424.

16. Shridhar, K.; Laumann, F.; Liwicki, M. A comprehensive guide to bayesian convolutional neural network with variational inference. arXiv 2019, arXiv:1901.02731.

17. Cheng, Y.; Wang, D.; Zhou, P.; Zhang, T. A survey of model compression and acceleration for deep neural networks. arXiv 2017, arXiv:1710.09282.

18. Willett, P. A review of chemical structure retrieval systems. J. Chemom. 1987, 1, 139-155. [CrossRef]

19. Willett, P. The calculation of molecular structural similarity: Principles and practice. Mol. Inform. 2014, 33, 403-413. [CrossRef] [PubMed]

20. Bajusz, D.; Rácz, A.; Héberger, K. Why is Tanimoto index an appropriate choice for fingerprint-based similarity calculations? J. Cheminformatics 2015, 7, 20. [CrossRef]

21. Cai, C.; Gong, J.; Liu, X.; Gao, D.; Li, H. Molecular similarity: Methods and performance. Chin. J. Chem. 2013, 31 , 1123-1132. [CrossRef]

22. Syuib, M.; Arif, S.M.; Malim, N. Comparison of similarity coefficients for chemical database retrieval. In Proceedings of the 2013 1st International Conference on Artificial Intelligence, Modelling and Simulation, Kota Kinabalu, Malaysia, 3-5 December 2013; pp. 129-133.

23. Willett, P. Textual and chemical information processing: Different domains but similar algorithms. Inf. Res. $2000,5,2$.

24. Abdo, A. Similarity-Based Virtual Screening Using Bayesian Inference Network; Universiti Teknologi Malaysia: Johor, Malaysia, 2009; Volume 3, p. 1.

25. Ahmed, A.; Abdo, A.; Salim, N. Ligand-based virtual screening using Bayesian inference network and reweighted fragments. Sci. World J. 2012, 2012, 410914. [CrossRef]

26. Abdelrahim, A.; Ahmed, A. Fragment Reweighting in Ligand-based Virtual Screening. Ph.D. Thesis, Universiti Teknologi Malaysia, Skudai, Malaysia, 2013.

27. Ahmed, A.; Salim, N.; Abdo, A. Fragment reweighting in ligand-based virtual screening. Adv. Sci. Lett. 2013, 19, $2782-2786$. [CrossRef]

28. Aldabagh, M.M. Quantium Inspired Probability Approaches in Ligend-Based Vitual Screen; UTM University: Johor, Malaysia, 2017.

29. Himmat, M.H.I. New Similarity Measures for Ligand-Based Virtual Screening; Universiti Teknologi Malaysia: Johor, Malaysia, 2017.

30. Nasser, M.; Salim, N.; Hamza, H. Molecular Similarity Searching Based on Deep Belief Networks with Different Molecular Descriptors. In Proceedings of the 2020 2nd International Conference on Big Data Engineering and Technology, Johor, Malaysia, 3-5 January 2020; pp. 18-24.

31. Nasser, M.; Salim, N.; Hamza, H.; Saeed, F.; Rabiu, I. Improved Deep Learning Based Method for Molecular Similarity Searching Using Stack of Deep Belief Networks. Molecules 2021, 26, 128. [CrossRef]

32. Mueller, J.; Thyagarajan, A. Siamese recurrent architectures for learning sentence similarity. In Proceedings of the AAAI Conference on Artificial Intelligence, Vancouver, BC, Canada, 22 February-1 March 2016.

33. Kohli, N. Automatic Kinship Verification in Unconstrained Faces Using Deep Learning; West Virginia University: Morgantown, WV, USA, 2019. 
34. Dhami, D.S.; Yan, S.; Kunapuli, G.; Page, D.; Natarajan, S. Beyond Textual Data: Predicting Drug-Drug Interactions from Molecular Structure Images using Siamese Neural Networks. arXiv 2019, arXiv:1911.06356.

35. Jeon, M.; Park, D.; Lee, J.; Jeon, H.; Ko, M.; Kim, S.; Choi, Y.; Tan, A.-C.; Kang, J. ReSimNet: Drug response similarity prediction using Siamese neural networks. Bioinformatics 2019, 35, 5249-5256. [CrossRef] [PubMed]

36. Louizos, C.; Ullrich, K.; Welling, M. Bayesian compression for deep learning. In Proceedings of the Advances in Neural Information Processing Systems, Long Beach, CA, USA, 4-9 December 2017; pp. 3288-3298.

37. Zhao, C.; Ni, B.; Zhang, J.; Zhao, Q.; Zhang, W.; Tian, Q. Variational convolutional neural network pruning. In Proceedings of the IEEE/CVF Conference on Computer Vision and Pattern Recognition, Long Beach, CA, USA, 15 June 2019; pp. $2780-2789$.

38. Salim, N.; Holliday, J.; Willett, P. Combination of fingerprint-based similarity coefficients using data fusion. J. Chem. Inf. Comput. Sci. 2003, 43, 435-442. [CrossRef]

39. Molchanov, P.; Tyree, S.; Karras, T.; Aila, T.; Kautz, J. Pruning convolutional neural networks for resource efficient inference. arXiv 2016, arXiv:1611.06440.

40. MDL Drug Data Report (MDDR). Accelrys Inc: San Diego, CA, USA. Available online: http:/ /www.accelrys.com (accessed on 15 January 2020).

41. Rohrer, S.G.; Baumann, K. Maximum unbiased validation (MUV) data sets for virtual screening based on PubChem bioactivity data. J. Chem. Inf. Model. 2009, 49, 169-184. [CrossRef] [PubMed]

42. Huang, N.; Shoichet, B.K.; Irwin, J.J. Benchmarking sets for molecular docking. J. Med. Chem. 2006, 49, 6789-6801. [CrossRef]

43. Cross, S.; Baroni, M.; Carosati, E.; Benedetti, P.; Clementi, S. FLAP: GRID molecular interaction fields in virtual screening. validation using the DUD data set. J. Chem. Inf. Model. 2010, 50, 1442-1450. [CrossRef] [PubMed]

44. Barker, E.J.; Buttar, D.; Cosgrove, D.A.; Gardiner, E.J.; Kitts, P.; Willett, P.; Gillet, V.J. Scaffold hopping using clique detection applied to reduced graphs. J. Chem. Inf. Model. 2006, 46, 503-511. [CrossRef] [PubMed]

45. Hert, J.; Willett, P.; Wilton, D.J.; Acklin, P.; Azzaoui, K.; Jacoby, E.; Schuffenhauer, A. Enhancing the effectiveness of similarity-based virtual screening using nearest-neighbor information. J. Med. Chem. 2005, 48, 7049-7054. [CrossRef]

46. Kogej, T.; Engkvist, O.; Blomberg, N.; Muresan, S. Multifingerprint based similarity searches for targeted class compound selection. J. Chem. Inf. Model. 2006, 46, 1201-1213. [CrossRef]

47. Nasser, M.; Salim, N.; Hamza, H.; Saeed, F. Deep Belief Network for Molecular Feature Selection in Ligand-Based Virtual Screening. In Proceedings of the International Conference of Reliable Information and Communication Technology, Kuala Lumpur, Malaysia, 23-24 July 2018; pp. 3-14.

48. Wilton, D.J.; Harrison, R.F.; Willett, P.; Delaney, J.; Lawson, K.; Mullier, G. Virtual screening using binary kernel discrimination: Analysis of pesticide data. J. Chem. Inf. Model. 2006, 46, 471-477. [CrossRef] [PubMed]

49. Legendre, P. Species associations: The Kendall coefficient of concordance revisited. J. Agric. Biol. Environ. Stat. 2005, 10, 226. [CrossRef] 